\title{
APPROXIMATELY MULTIPLICATIVE MAPS FROM WEIGHTED SEMILATTICE ALGEBRAS
}

\author{
YEMON CHOI \\ (Received 30 March 2012; accepted 30 March 2013; first published online 17 June 2013) \\ Communicated by G. Willis
}

\begin{abstract}
We investigate which weighted convolution algebras $\ell_{\omega}^{1}(S)$, where $S$ is a semilattice, are AMNM in the sense of Johnson ['Approximately multiplicative functionals', J. Lond. Math. Soc. (2) 34(3) (1986), 489-510]. We give an explicit example where this is not the case. We show that the unweighted examples are all AMNM, as are all $\ell_{\omega}^{1}(S)$ where $S$ has either finite width or finite height. Some of these finite-width examples are isomorphic to function algebras studied by Feinstein ['Strong Ditkin algebras without bounded relative units', Int. J. Math. Math. Sci. 22(2) (1999), 437-443]. We also investigate when $\left(\ell_{\omega}^{1}(S), \mathbb{M}_{2}\right)$ is an AMNM pair in the sense of Johnson ['Approximately multiplicative maps between Banach algebras', J. Lond. Math. Soc. (2) 37(2) (1988), 294-316], where $\mathbb{M}_{2}$ denotes the algebra of $2 \times 2$ complex matrices. In particular, we obtain the following two contrasting results: (i) for many nontrivial weights on the totally ordered semilattice $\mathbb{N}_{\min }$, the pair $\left(\ell_{\omega}^{1}\left(\mathbb{N}_{\min }\right), \mathbb{M}_{2}\right)$ is not AMNM; (ii) for any semilattice $S$, the pair $\left(\ell^{1}(S), \mathbb{M}_{2}\right)$ is AMNM. The latter result requires a detailed analysis of approximately commuting, approximately idempotent $2 \times 2$ matrices.
\end{abstract}

2010 Mathematics subject classification: primary 39B72; secondary 46J10.

Keywords and phrases: AMNM, approximate homomorphism, Feinstein algebra, semilattice, weighted convolution algebra.

\section{Introduction}

1.1. Setting the scene. Given a constant $\delta>0$, we say that a functional $\psi$ on a Banach algebra $A$ is $\delta$-multiplicative if the bilinear map $(a, b) \mapsto \psi(a) \psi(b)-\psi(a b)$ has norm at most $\delta$. It is convenient, thinking of $\delta$ as small, to call such functionals approximately multiplicative or almost multiplicative (we shall use the former phrase). Approximately multiplicative functionals have been studied by several authors: an obvious way to obtain examples is to take a multiplicative functional and add a functional of small norm, thought of as a perturbation. The question naturally arises as to whether all approximately multiplicative functionals occur in this way.

In [10], Johnson undertook a systematic study of this phenomenon, and coined the acronym AMNM (for 'approximately multiplicative implies near multiplicative'). The precise definition will be deferred to a later section. Many examples of commutative

(c) 2013 Australian Mathematical Publishing Association Inc. 1446-7887/2013 \$16.00 
Banach algebras with the AMNM property are given in [10], as are some basic hereditary properties. See also [9, 14] for results on uniform algebras, and [7] for results on certain nonuniform function algebras, including $C^{k}[0,1]^{m}$. Johnson [11] widens the scope of the problem by considering not just functionals, but approximately multiplicative linear maps between given Banach algebras. This leads to the notion of an AMNM pair; again, the precise definition will be given below. We note that, as a special case of [11, Theorem 3.1], every amenable Banach algebra has the AMNM property; however, several of the examples in $[7,9,10]$ possess nonzero point derivations, so that amenability is far from necessary for AMNM.

In this paper, we investigate these AMNM problems for the weighted $\ell^{1}$ convolution algebras of semilattices. Such algebras have provided provide useful test cases for various conjectures and techniques concerning commutative Banach algebras. Moreover, any weighted semilattice algebra contains a dense subalgebra spanned by commuting idempotents. Thus, some of our work can be viewed as continuing an old strand of Banach algebra theory, which considers lifting and perturbation questions for families of idempotents. The main difference here is that we are not limiting ourselves to families of pairwise orthogonal idempotents, but allowing more complicated order structure.

The original motivation for the present work arises from studying the cases where the underlying semilattice is $\mathbb{N}_{\text {min }}$, the set of natural numbers equipped with pairwise minimum as a semigroup product. The weighted $\ell^{1}$-algebras of $\mathbb{N}_{\text {min }}$ turn out to be isomorphic to function algebras that were studied by Feinstein in [3]; they have also been studied in the context of certain generalized notions of amenability; see, for instance, [2, Section 3.10]. Moreover, some of these algebras satisfy such versions of amenability while having nontrivial second-degree simplicial cohomology (the present author, unpublished calculations).

1.2. Overview of the paper. We have tried to make this paper self-contained, save for some basic knowledge of Banach algebras. Thus, in Section 2 we give the relevant definitions of the AMNM property for algebras and for pairs of algebras, as promised earlier; and we record some basic observations on convolution algebras and their characters. We then observe that $\ell^{1}(S)$ is AMNM for any semilattice $S$ (Theorem 3.1). On the other hand, we give an explicit example of a semilattice $T$ and a weight on $T$ such that the weighted convolution algebra $\ell_{\omega}^{1}(T)$ is not AMNM (Theorem 3.4). In Section 3.3, as a special case of a general technical result, we prove that if $S$ has either finite width or finite height, then $\ell_{\omega}^{1}(S)$ is AMNM for every weight $\omega$. This applies in particular when $S=\mathbb{N}_{\text {min }}$, the original case of interest.

The picture is far less complete if we consider approximately multiplicative maps into algebras other than $\mathbb{C}$. Let $\mathbf{T}_{2}$ be the (commutative, nonsemisimple) algebra of dual numbers over $\mathbb{C}$, and let $\mathbb{M}_{2}$ be the (noncommutative, semisimple) algebra of $2 \times 2$ matrices with entries in $\mathbb{C}$. In Theorem 4.2 we show that whenever $\omega$ is a nontrivial weight on $\mathbb{N}_{\text {min }}$, then $\left(\ell_{\omega}^{1}\left(\mathbb{N}_{\text {min }}\right), \mathbf{T}_{2}\right)$ is not an AMNM pair. In Theorem 4.7 we show that for many nontrivial weights on $\mathbb{N}_{\text {min }}$, the pair $\left(\ell_{\omega}^{1}\left(\mathbb{N}_{\min }\right), \mathbb{M}_{2}\right)$ fails to be AMNM. 
These examples suggest that, if we want positive AMNM results for range algebras other than $\mathbb{C}$, we should focus attention on the unweighted case. Indeed, we prove that for an arbitrary semilattice $S$, the pairs $\left(\ell^{1}(S), \mathbf{T}_{2}\right)$ and $\left(\ell^{1}(S), \mathbb{M}_{2}\right)$ are 'uniformly AMNM' (the terminology is explained below in Definition 2.4). The proof of this for $\mathbb{M}_{2}$ takes up all of Section 5: although the techniques used are elementary, a complete proof seems to require substantially more work than is needed for $\mathbf{T}_{2}$. Finally, we close the paper by briefly discussing some possible avenues for future work.

REMARK 1.1. Some of our calculations would work for certain weighted algebras on abelian Clifford semigroups, specifically those where the weight is trivial on each group component. We have decided to consider only the semilattice case for now: an adequate treatment of weighted abelian Clifford semigroups would require a more detailed look at AMNM problems for Beurling algebras than the present paper can accommodate. The same remarks apply for inverse semigroups: we did not see how to go beyond superficial generalizations of the results here.

Remark 1.2. After completing the work presented in this paper, we learned of the paper [12], which considers a related but different notion of AMNM (briefly, the order of quantifiers is different). Although the stability result that is proved in [12, Theorems 2 and 5] is different from ours, it may be possible to use those arguments to streamline the approach taken in Section 5, and perhaps even to extend the results of Section 5 from $\mathbb{M}_{2}$ to $\mathbb{M}_{n}$.

\section{Preliminaries}

We assume familiarity with the basics of Banach spaces and Banach algebras. Throughout $\widehat{\otimes}$ denotes the projective tensor product of Banach spaces. If $A$ is a Banach algebra then $\pi_{A}: A \widehat{\otimes} A \rightarrow A$ denotes the unique bounded bilinear map satisfying $\pi_{A}\left(a_{1} \otimes a_{2}\right)=a_{1} a_{2}$.

2.1. Defining AMNM. Our notation is different from that of Johnson's papers $[10,11]$, and so we repeat some of the basic definitions for the sake of clarity.

Definition 2.1 (Multiplicative defect). Let $A$ and $B$ be Banach algebras, and let $T$ : $A \rightarrow B$ be a bounded linear map. We define the multiplicative defect of $T$ to be

$$
\begin{aligned}
\operatorname{def}(T) & =\left\|T \circ \pi_{A}-\pi_{B} \circ(T \otimes T): A \widehat{\otimes} A \rightarrow B\right\| \\
& =\sup \{\|T(x y)-T(x) T(y)\|: x, y \in A,\|x\| \leq 1,\|y\| \leq 1\} .
\end{aligned}
$$

$T$ is said to be $\delta$-multiplicative, in the sense of [11], when $\operatorname{def}(T) \leq \delta$. Note that Johnson uses the notation $T^{\vee}$ instead of $\operatorname{def}(T)$. Of course, a 0 -multiplicative map is just one that is multiplicative in the usual sense, that is, a continuous linear algebra homomorphism (which need not be unital, even if $A$ and $B$ are unital algebras). We denote by $\operatorname{Mult}(A, B)$ the set of multiplicative, bounded linear maps $A \rightarrow B$; a nonzero element of $\operatorname{Mult}(A, \mathbb{C})$ is called a character of $A$.

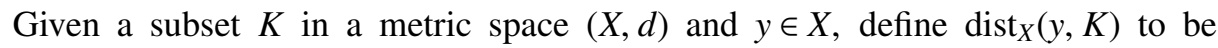
$\inf _{x \in X} d(x, y)$. 
Definition 2.2 (AMNM algebras, [10]). A Banach algebra $A$ is said to be $A M N M$, or have the AMNM property, or have stable characters, if for each $\varepsilon>0$ there exists $\delta>0$ such that

$$
\psi \in A^{*}, \quad \operatorname{def}(\psi) \leq \delta \Longrightarrow \operatorname{dist}_{A^{*}}(\psi, \operatorname{Mult}(A, \mathbb{C})) \leq \varepsilon .
$$

While Definition 2.2 does not require $A$ to be commutative, it seems most natural to study the AMNM property for commutative Banach algebras, since those are the ones for which characters are most informative (via Gelfand theory). For noncommutative algebras $A$, the following definition seems more natural.

Definition 2.3 (AMNM pairs of Banach algebras, [11]). Let $A$ and $B$ be Banach algebras. We say that the pair $(A, B)$ is an $A M N M$ pair if, for every $K>0$ and $\varepsilon>0$, there exists $\delta>0$ such that

$$
T \in \mathcal{L}(A, B), \quad\|T\| \leq K, \quad \operatorname{def}(T) \leq \delta \Longrightarrow \operatorname{dist}_{\mathcal{L}(A, B)}(T, \operatorname{Mult}(A, B)) \leq \varepsilon .
$$

The presence of the a priori upper bound $K$ may seem curious at first sight. One reason for imposing such a bound is that we can find $T$ such that $\operatorname{dist}_{\mathcal{L}(A, B)}(T$, Mult $(A, B))$ is arbitrarily small while $\operatorname{def}(T) \geq 1$. See [11, page 295] for an example with $A=\mathbb{C}$ and $B$ the algebra $\mathbb{M}_{2}$ of $2 \times 2$ matrices.

Nevertheless, in the present paper, we shall obtain examples of pairs of algebras which are not just AMNM, but which satisfy a stronger property, defined as follows.

Definition 2.4. Let $A$ and $B$ be Banach algebras. We say that $(A, B)$ is a uniformly $A M N M$ pair if, for every $\varepsilon>0$, there exists $\delta>0$ such that

$$
T \in \mathcal{L}(A, B), \quad \operatorname{def}(T) \leq \delta \Longrightarrow \operatorname{dist}_{\mathcal{L}(A, B)}(T, \operatorname{Mult}(A, B)) \leq \varepsilon .
$$

With this terminology, a Banach algebra $A$ is AMNM if and only if the pair $(A, \mathbb{C})$ is uniformly AMNM. In some cases, AMNM pairs are automatically uniformly AMNM: for instance, this is the case when $B=C(X)$, since a $\delta$-multiplicative linear map $A \rightarrow C(X)$ has norm at most $1+\delta[8$, Proposition 5.5]. Note that Johnson [11, Example 1.5] has given an example of a commutative, semisimple Banach algebra $B$ for which the pair $(\mathbb{C}, B)$ is AMNM but not uniformly AMNM.

2.2. Some notation and terminology. A weight on a set $S$ is a function $\omega: S \rightarrow$ $(0, \infty)$. Given such a weight $\omega$, we write $\ell_{\omega}^{1}(S)$ for the corresponding weighted $\ell^{1}$ space. Throughout this paper, whenever we refer to a weight on a semigroup, we always mean a submultiplicative weight. By a weighted semigroup we mean a pair $(S, \omega)$ where $S$ is a semigroup and $\omega$ is a weight on $S$. A routine calculation shows that if $(S, \omega)$ is a weighted semigroup, then $\ell_{\omega}^{1}(S)$, equipped with the natural convolution product, is a Banach algebra, called the weighted semigroup algebra or convolution algebra of $(S, \omega)$.

A bounded function $f$ from a set $S$ to a Banach space $B$ has a unique continuous extension to a bounded linear map $\ell^{1}(S) \rightarrow B$, whose norm is precisely $\sup _{t \in S}\|f(t)\|$. Moreover, if $S$ is a semigroup and $B$ is a Banach algebra, this 
extension will be multiplicative if and only if the original function $f$ is multiplicative. Thus, the characters of $\ell^{1}(S)$ are in natural bijection with the nonzero semigroup homomorphisms $S \rightarrow(\mathbb{C}, \times)$, sometimes called the semi-characters of $S$.

The corresponding version for weighted semigroup algebras is equally straightforward: bounded linear maps $\ell_{\omega}^{1}(S) \rightarrow B$ correspond to functions $f: S \rightarrow B$ such that

$$
\sup _{s \in S} \omega(s)^{-1}\|f(s)\|<\infty,
$$

and so forth. Such a function will be called an $\omega$-bounded map from $S$ to $B$. In the case where $B=\mathbb{C}$ and $f: S \rightarrow \mathbb{C}$, the quantity in (2.1) will be denoted by $\|f\|_{\infty, \omega^{-1}}$.

Since we can naturally and isometrically identify $\ell_{\omega}^{1}(S) \widehat{\otimes} \ell_{\omega}^{1}(S)$ with $\ell_{\omega \times \omega}^{1}(S \times S)$, it is easy to check that for a given function $f: S \rightarrow B$, the multiplicative defect of the corresponding linear map $\ell_{\omega}^{1}(S) \rightarrow B$ is

$$
\operatorname{def}_{\omega}(f):=\sup _{x, y \in S} \frac{\|f(x) f(y)-f(x y)\|}{\omega(x) \omega(y)} .
$$

In this way, all AMNM questions where the domain algebra $A$ is a weighted semigroup algebra can be rephrased in terms of $\omega$-bounded maps from the given semigroup and $(\omega \times \omega)$-bounded maps from its Cartesian square. This is sometimes convenient if we need to define a map via case-by-case checking.

2.3. Weighted semilattice algebras and their characters. A semilattice is a commutative semigroup in which each element is idempotent. Even quite simple semilattices can give rise to interesting Banach algebras, once we allow for weights. The following examples provided the initial motivation for this paper, and will be revisited in Theorems 4.2 and 4.7 .

ExAmple 2.5 ('Feinstein algebras'). Let $\mathbb{N}_{\min }$ denote the semilattice obtained by equipping the set of natural numbers with the binary operation $(m, n) \mapsto \min (m, n)$. The weights on this semilattice are precisely the functions $\omega: \mathbb{N} \rightarrow[1, \infty)$. The convolution algebra $\ell_{\omega}^{1}\left(\mathbb{N}_{\min }\right)$ is semisimple and its character space can be naturally identified with $\mathbb{N}$. (This is alluded to, without details, in [5, 11.1.5]; see also Lemma 2.8 below.) In fact, the Gelfand transform maps $\ell_{\omega}^{1}\left(\mathbb{N}_{\min }\right)$ onto a dense subalgebra $B_{\omega} \subset c_{0}(\mathbb{N})$, defined by

$$
B_{\omega}=\left\{f \in c_{0}(\mathbb{N}):\left|f_{1}\right| \omega(1)+\sum_{j \geq 2}\left|f_{j+1}-f_{j}\right| \omega(j)<\infty\right\}
$$

equipped with the obvious norm. The unitizations of the algebras $B_{\omega}$ are isomorphic to examples studied in [3]. Note that in the cases where $\omega$ is bounded, $\ell_{\omega}^{1}\left(\mathbb{N}_{\min }\right)$ is isomorphic as a Banach algebra to $\ell^{1}\left(\mathbb{N}_{\min }\right)$, and $B_{\omega}$ will consist of those $c_{0}$-sequences which have bounded variation. For more details, see [2, Section 3].

REMARK 2.6. In the literature, terminology and notation for these examples has varied. Strictly speaking, Feinstein's construction in [3] is more general: he defines, for any 
given sequence $\alpha=\left(\alpha_{n}\right)_{n \geq 1}$ of strictly positive real numbers, a commutative unital Banach algebra $A_{\alpha} \subset C(\mathbb{N} \cup\{\infty\})$, and in his notation our $B_{\alpha}$ coincides with $A_{\alpha} \cap c_{0}$. Note, however, that in some later papers the algebra $A_{\alpha}$ is defined to be what we have denoted by $B_{\alpha}$.

For general semilattices, a systematic approach to the character space is via the language of filters. First we introduce some notation that will be used later in Section 3.3. Given a semilattice $S$ and a subset $E \subseteq S$, we define the following sets:

$$
\langle E\rangle_{n}=\left\{x_{1} \cdots x_{n}: x_{1}, \ldots, x_{n} \in E\right\} \quad(n \geq 1),
$$

and $\langle E\rangle=\bigcup_{n \geq 1}\langle E\rangle_{n}$. Note that $\langle E\rangle$ is the subsemilattice of $S$ generated by $E$.

Definition 2.7. Let $S$ be a semilattice and let $F \subseteq S$. We say that $F$ is a filter in $S$ if it satisfies the following three properties: it is nonempty; it is closed under multiplication (that is, $x y \in F$ whenever $x, y \in F$ ); and it is upwards closed in $S$ (that is, whenever $x \in S$ and $y \in F$ with $x y \in F$, then $x \in F$ ).

Given a nonempty subset $E$ in a semilattice $S$, there exists a smallest filter in $S$ which contains $E$. This can be described concretely: it is the set

$$
F=\bigcup_{y \in\langle E\rangle}\{x \in S: x \geq y\} .
$$

The following lemma assembles some basic results, which are well known, at least implicitly, for multiplicative linear functions on semilattice algebras. (See, for example, $[5,11.1 .1]$.) Since the proofs are no harder for the weighted versions, we leave them to the reader.

Lemma 2.8. Let $S$ be a semilattice and $F \subset S$. The following are equivalent:

(i) $F$ is a filter in $S$;

(ii) the indicator function of $F$ is a character on $\ell_{\omega}^{1}(S)$, for every submultiplicative weight $\omega$;

(iii) there exists a submultiplicative weight $\omega$ such that the indicator function of $F$ is a character on $\ell_{\omega}^{1}(S)$.

Moreover, if $\phi: S \rightarrow \mathbb{C}$ is multiplicative and nonzero, and $\phi=1$ on some subset $E \subseteq S$, then $\phi=1$ on the filter generated by $E$.

\section{The AMNM property for weighted semilattice algebras}

\subsection{The unweighted case, as a guide}

Theorem 3.1. Let $S$ be a semilattice. Then $\ell^{1}(S)$ is AMNM.

This result, or equivalent reformulations, may have been implicitly known to previous authors. We shall give a complete proof, since it allows us to introduce some basic results and techniques which will be instructive for later arguments. 
Define $f:[0,1 / 4] \rightarrow[0,1 / 2]$ by

$$
f(t)=\frac{1}{2}(1-\sqrt{1-4 t}) .
$$

and define $\rho(t)=t^{-1} f(t)$ for $0<t \leq 1 / 4$. The following properties are straightforward to verify:

- $\quad f$ is convex and monotone increasing, and $f(0)=0$;

- $\quad \rho$ is monotone increasing on $(0,1 / 4]$, and $\lim _{t \searrow 0} \rho(t)=1$.

Notation. Given $r \geq 0$ and $w \in \mathbb{C}$, denote the closed disc of radius $r$ and centre $w$ by $\overline{\mathbb{D}}_{w}(r)$. If $r>0$, denote the open disc of radius $r$ and centre $w$ by $\mathbb{D}_{w}(r)$.

Lemma 3.2. Let $z \in \mathbb{C}$ and $\varepsilon \in[0,1 / 4)$. If $z^{2}-z \in \overline{\mathbb{D}}_{0}(\varepsilon)$, then $\operatorname{dist}_{\mathbb{C}}(z,\{0,1\}) \leq \rho(\varepsilon) \varepsilon$.

Proof. Put $w=z-z^{2}$. Then $\left(z-\frac{1}{2}\right)^{2}=\frac{1}{4}(1-4 w)$, and so $z-\frac{1}{2}= \pm \frac{1}{2} \sqrt{1-4 w}$ (taking the branch of the square root function for which $\sqrt{1-4 w} \rightarrow 1$ as $w \rightarrow 0$ ). It follows that

$$
\operatorname{dist}_{\mathbb{C}}(z,\{0,1\}) \leq\left|\frac{1}{2}-\frac{1}{2} \sqrt{1-4 w}\right| .
$$

The Taylor expansion (about 0 ) of the function $w \mapsto 1-\sqrt{1-4 w}$ has nonnegative coefficients. Hence $\operatorname{dist}_{\mathbb{C}}(z,\{0,1\}) \leq f(|w|)$, and the rest follows from our earlier observations.

Theorem 3.1 follows immediately from the following technical result.

Proposition 3.3. Let $S$ be a semilattice, and let $\psi: S \rightarrow \mathbb{C}$ satisfy $\operatorname{def}(\psi)<1 / 5$. Let

$$
S_{1}=\psi^{-1}\left(\mathbb{D}_{1}(7 / 25)\right)=\{e \in S:|\psi(e)-1|<7 / 25\},
$$

and let $\chi$ be the indicator function of $S_{1}$. Then $\chi$ is a multiplicative function $S \rightarrow \mathbb{C}$, satisfying

$$
\|\psi-\chi\|_{\infty} \leq \frac{7}{5} \operatorname{def}(\psi)
$$

Proof. We first note that

$$
\rho\left(\frac{1}{5}\right)=\frac{5}{2}\left(1-\sqrt{\frac{1}{5}}\right)=\frac{1}{2}(5-\sqrt{5})<\frac{1}{2}(5-2.2)=\frac{7}{5} .
$$

Hence, by Lemma 3.2,

$$
\sup _{e \in S} \min (|\psi(e)-1|,|\psi(e)|) \leq \sup _{e \in S} \frac{7}{5}\left|\psi(e)^{2}-\psi(e)\right|=\frac{7}{5} \operatorname{def}(\psi)<\frac{7}{25} .
$$

Set $S_{0}=\psi^{-1}\left(\mathbb{D}_{0}(7 / 25)\right)$. Then $S=S_{0} \sqcup S_{1}$, so that the previous inequality immediately yields $\|\psi-\chi\|_{\infty} \leq(7 / 5)\left\|\psi^{2}-\psi\right\|_{\infty}=(7 / 5) \operatorname{def}(\psi)($ see $(2.2))$.

To prove that $\chi$ is multiplicative, it suffices by Lemma 2.8 to show that $S_{1}$ is either empty or a filter. Suppose that $S_{1}$ is nonempty. If $e, f \in S_{1}$, then $|\psi(e) \psi(f)|>$ $(18 / 25)^{2}>1 / 2$. Hence

$$
|\psi(e f)|>\frac{1}{2}-\operatorname{def}(\psi)>\frac{1}{2}-\frac{1}{5}>\frac{7}{25},
$$


forcing $e f \in S \backslash S_{0}=S_{1}$. If $e \geq f \in S$ and $f \in S_{1}$, then since $|\psi(f)|^{-1}<25 / 18$,

$$
|\psi(e)-1| \leq \frac{25}{18}|\psi(e) \psi(f)-\psi(f)| \leq \frac{25}{18} \operatorname{def}(\psi)<\frac{5}{18}<\frac{7}{25},
$$

forcing $e \in S_{1}$. Thus $S_{1}$ is a filter, and the proof is complete.

\subsection{A weighted semilattice which is not AMNM.}

THEOREM 3.4. There exists a locally finite semilattice $T$ and a submultiplicative weight $\omega$ on $T$ such that $\ell_{\omega}^{1}(T)$ is not $A M N M$.

The counterexample $T$ will be built out of copies of free semilattices. Given a set $S$, let $\mathbf{2}_{*}^{[S]}$ denote the free semilattice generated by $S$; this can be identified with the set of all nonempty finite subsets of $S$, with semigroup product given by union. For our purposes it is more convenient to regard elements of $\mathbf{2}_{*}^{[S]}$ as reduced words in the generators. There is a natural length function $\gamma_{S}: \mathbf{2}_{*}^{[S]} \rightarrow \mathbb{N}$, where $\gamma_{S}(x)$ is the minimum number of elements in $S$ needed to generate $x$. A little thought shows that $\gamma_{S}(x y) \leq \min \left(|S|, \gamma_{S}(x)+\gamma_{S}(y)\right)$ for all $x, y \in \mathbf{2}_{*}^{[S]}$.

If $S$ is a finite set, then $\mathbf{2}_{*}^{[S]}$ has a zero (that is, minimum) element, namely the product of all elements of $S$; we denote this element by $\theta_{S}$. Note that $\gamma_{S}\left(\theta_{S}\right)=|S|$.

Proposition 3.5. Let $S$ be a finite set of cardinality at least 2 . Fix a constant $C>1$, and define $\omega_{S}: \mathbf{2}_{*}^{[S]} \rightarrow[1, \infty)$ by

$$
\omega_{S}(e)= \begin{cases}C^{\gamma_{S}(e)} & \text { if } e \neq \theta_{S} \\ C & \text { if } e=\theta_{S}\end{cases}
$$

Then $\omega_{S}$ is a submultiplicative weight on $\mathbf{2}_{*}^{[S]}$. Moreover, if we define $\psi: \mathbf{2}_{*}^{[S]} \rightarrow$ $\{0,1\}$ by

$$
\psi(e)= \begin{cases}1 & \text { if } e \neq \theta_{S}, \\ 0 & \text { if } e=\theta_{S},\end{cases}
$$

then:

(i) as an element of $\ell_{\omega_{S}}^{1}\left(\mathbf{2}_{*}^{[S]}\right)^{*}, \operatorname{def}(\psi) \leq C^{-|S|}$;

(ii) if $\phi: \mathbf{2}_{*}^{[S]} \rightarrow\{0,1\}$ is multiplicative, then

$$
\sup _{e \in \mathbf{2}_{*}^{[S]}} \omega_{S}(e)^{-1}|\psi(e)-\phi(e)| \geq C^{-1} .
$$

Proof. Since $\gamma_{S}$ is subadditive and $\omega_{S}(e) \leq C^{\gamma_{S}(e)}$ for all $e \in \mathbf{2}_{*}^{[S]}$, it is clear that $\omega_{S}$ is submultiplicative.

To prove (i), let $e, f \in \mathbf{2}_{*}^{[S]}$. If $e f \neq \theta_{S}$ then $\psi(e) \psi(f)=1=\psi(e f)$. If $e=\theta_{S}$ or $f=\theta_{S}$ then $\psi(e) \psi(f)=0=\psi(e f)$. The only remaining cases are those where $e, f \neq \theta_{S}$ while $e f=\theta_{S}$. In such cases, we have $\gamma_{S}(e)+\gamma_{S}(f) \geq|S|$, and so

$$
|\psi(e) \psi(f)-\psi(e f)|=1 \leq \delta C^{-|S|} \omega(e) \omega(f) .
$$

Thus in all cases, $|\psi(e) \psi(f)-\psi(e f)| \leq C^{-|S|} \omega(e) \omega(f)$. 
To prove (ii), let $\phi: \mathbf{2}_{*}^{[S]} \rightarrow\{0,1\}$ be multiplicative. If $\phi\left(\theta_{S}\right)=1$, then

$$
\omega\left(\theta_{S}\right)^{-1}\left|\phi\left(\theta_{S}\right)-\psi\left(\theta_{S}\right)\right|=C^{-1}
$$

If not, then $\phi\left(\theta_{S}\right)=0$. Therefore, since $\phi$ is multiplicative and $S$ generates $\boldsymbol{2}_{*}^{[S]}$, there exists $s_{0} \in S$ with $\phi\left(s_{0}\right)=0$, and so

$$
\omega\left(s_{0}\right)^{-1}\left|\phi\left(s_{0}\right)-\psi\left(s_{0}\right)\right|=C^{-1} .
$$

If $\left(F_{i}\right)_{i \in \mathbb{I}}$ is a family of semilattices, consider the set $\{\theta\} \sqcup \bigsqcup_{i \in \mathbb{I}} F_{i}$, where $\theta$ is a formal symbol. This can be made into a semilattice if we define the product as follows: $\theta$ is an absorbing zero element; the product of two elements in $F_{i}$ is their usual product; the product of elements in $F_{i}$ and $F_{j}$ is $\theta$ whenever $i \neq j$. We call this semilattice the orthogonal direct sum of the family $\left(F_{i}\right)_{i \in \mathbb{I}}$.

Proof of Theorem 3.4. Let $\left(F_{n}\right)_{n \geq 1}$ be a sequence of finite sets with $\left|F_{n}\right| \nearrow \infty$. Define $T$ to be the orthogonal direct sum of the family $\left(2_{*}^{\left[F_{n}\right]}\right)_{n \in \mathbb{N}}$, with $\theta$ being the zero element of $T$. To ease notation, denote the zero element of $\boldsymbol{2}_{*}^{\left[F_{n}\right]}$ by $\theta_{n}$, and denote the length function of $\boldsymbol{2}_{*}^{\left[F_{n}\right]}$ by $\gamma_{n}$.

Fix a constant $C>1$, and define $\omega: T \rightarrow[1, \infty)$ by

$$
\omega(\theta):=1, \quad \omega\left(\theta_{n}\right):=C \text { for each } n \in \mathbb{N}, \quad \omega(e):=C^{\gamma_{n}(e)} \text { if } e \in \mathbf{2}_{*}^{\left[F_{n}\right]} \backslash\left\{\theta_{n}\right\} .
$$

Define $\psi_{n}: T \rightarrow\{0,1\}$ by

$$
\psi_{n}(e)= \begin{cases}1 & \text { if } E \in S_{n} \backslash\left\{\theta_{n}\right\} \\ 0 & \text { otherwise }\end{cases}
$$

If $\phi: T \rightarrow\{0,1\}$ is multiplicative, then by part (ii) of Proposition 3.5,

$$
\left\|\psi_{n}-\phi\right\| \geq \sup \left\{\omega(e)^{-1}\left|\psi_{n}(e)-\phi(e)\right|: e \in \mathbf{2}_{*}^{\left[F_{n}\right]}\right\} \geq C^{-1} .
$$

Hence,

$$
\operatorname{dist}_{\ell_{\omega}^{1}(T)^{*}}\left(\psi_{n}, \operatorname{Mult}\left(\ell_{\omega}^{1}(T), \mathbb{C}\right)\right) \geq C^{-1} \quad \text { for all } n .
$$

On the other hand, by Proposition 3.5(i),

$$
\operatorname{def}_{\omega}\left(\psi_{n}\right)=\sup \left\{\frac{\left|\psi_{n}(e) \psi_{n}(f)-\psi_{n}(e f)\right|}{\omega(e) \omega(f)}: e, f \in \mathbf{2}_{*}^{\left[F_{n}\right]}\right\} \leq C^{-\left|F_{n}\right|} \rightarrow 0 .
$$

Thus $\ell_{\omega}^{1}(T)$ is not AMNM.

The example $(T, \omega)$ was found while trying to prove that all weighted semilattice algebras are AMNM, and realizing that the attempted proof only worked when one could verify a certain technical condition on a given weighted semilattice $(S, \omega)$. This condition, and the proof that it suffices to ensure that $\ell_{\omega}^{1}(S)$ is AMNM, will be our next topic. 
3.3. Weighted semilattices which are AMNM. The following lemma is a substitute for Lemma 3.2. It is less informative in the case of $\ell^{1}(S)$, but is more convenient in weighted cases.

Lemma 3.6. Let $A$ be a Banach algebra, e an idempotent in $A$, and $\psi \in A^{*}$. Then

$$
\min (|\psi(e)|,|1-\psi(e)|) \leq \operatorname{def}(\psi)^{1 / 2}\|e\|
$$

Proof. If $e=0$ this is trivial. If $e \neq 0$, observe that

$$
\operatorname{def}(\psi) \geq \frac{\left|\psi(e)-\psi(e)^{2}\right|}{\|e\|^{2}}=\frac{|\psi(e)|}{\|e\|} \frac{|1-\psi(e)|}{\|e\|} \geq\left(\frac{\min (|\psi(e)|,|1-\psi(e)|)}{\|e\|}\right)^{2},
$$

and (3.3) follows.

By (3.3) and the definition of the norm on $\ell_{\omega}^{1}(S)$, we see that if $\psi: S \rightarrow \mathbb{C}$ is $\omega$-bounded with $\operatorname{def}_{\omega}(\psi) \leq \delta$, then there exists an $\omega$-bounded function $\phi: S \rightarrow\{0,1\}$ such that $\|\psi-\phi\|_{\infty, \omega^{-1}} \leq \delta^{1 / 2}$. Since

$$
\begin{aligned}
|\phi(s) \phi(t)-\phi(s t)| & \leq\left\{\begin{array}{r}
|\phi(s)||\phi(t)-\psi(t)|+|\phi(s)-\psi(s)||\psi(t)| \\
+|\psi(s) \psi(t)-\psi(s t)|+|\psi(s t)-\phi(s t)|
\end{array}\right. \\
& \leq 1 \cdot \delta^{1 / 2} \omega(t)+\delta^{1 / 2} \omega(s) \cdot\left(1+\delta^{1 / 2} \omega(t)\right)+\delta \omega(s) \omega(t)+\delta^{1 / 2} \omega(s t) \\
& =\delta^{1 / 2} \omega(t)+\delta^{1 / 2} \omega(s)+2 \delta \omega(s) \omega(t)+\delta^{1 / 2} \omega(s t),
\end{aligned}
$$

we have $\operatorname{def}_{\omega}(\phi) \leq 3 \delta^{1 / 2}+2 \delta$. From this, routine arguments (which we omit) yield the following necessary and sufficient condition for $\ell_{\omega}^{1}(S)$ to be AMNM.

Corollary 3.7. Let $(S, \omega)$ be a weighted semilattice. The following are equivalent:

(i) $\ell_{\omega}^{1}(S)$ is AMNM;

(ii) for any $\varepsilon>0$, there exists $\delta>0$, such that whenever $\phi: S \rightarrow\{0,1\}$ satisfies

$$
\sup _{e, f \in S} \frac{|\phi(e f)-\phi(e) \phi(f)|}{\omega(e) \omega(f)} \leq \delta
$$

then there exists a subset $F \subseteq S$, either empty or a filter, such that

$$
\sup _{e \in S} \frac{\left|\chi_{F}(e)-\phi(e)\right|}{\omega(e)} \leq \varepsilon
$$

It turns out that a certain natural, structural condition on a semilattice $S$ will imply that $\ell_{\omega}^{1}(S)$ is AMNM for any choice of weight function $\omega$. This condition was rediscovered by the author in the course of the present investigations; it was subsequently pointed out (personal communication; see [13]) that it was already known by a standard name in the lattice-theoretic literature. 
Definition 3.8 (The breadth of a semilattice). Let $E$ be a subset of a semilattice $S$. We define

$$
b_{\text {loc }}(E)=\inf \left\{n \in \mathbb{N}:\langle E\rangle_{n}=\langle E\rangle\right\},
$$

with the usual convention that the infimum of the empty set is $+\infty$. The breadth of $S$ is defined to be $\sup _{E \subseteq S} b_{\text {loc }}(E)$, and is denoted by $b(S)$.

Here, the sets $\langle E\rangle_{n}$ and $\langle E\rangle$ are as defined in (2.3). Note that having finite breadth is not the same as being finitely generated as a semigroup. Indeed, finitely generated semilattices are finite.

The following examples of semilattices with finite breadth are presumably well known to specialists, but since we were unable to find explicit references in the literature, details of the proofs are included. (For some discussion, see, for example, [1, Section IV.10].)

Example 3.9. Let $S$ be the free semilattice on $n$ generators. Then $b(S)=n$.

Proof. Since $S$ has height $n$, it will follow from Example 3.11 below that $b(S) \leq n$. On the other hand, if $F$ denotes the set of generators of $S$, then $\langle F\rangle_{n}=S$ while $\langle F\rangle_{n-1}$ does not contain the minimal element of $S$, implying that $b(S) \geq b_{\text {loc }}(F)>n-1$.

EXAMPLE 3.10. If $S$ has width $\leq n$ as a partially ordered set (that is, there exist $n$ chains in $S$ whose union is all of $S$ ) then $b(S) \leq n$.

Proof. Fix chains $C_{1}, \ldots, C_{n}$ in $S$ such that $S=\bigcup_{i=1}^{n} C_{i}$. Let $E \subseteq S$ and $x \in\langle E\rangle$. For some integer $k$ there exists $y_{1}, \ldots, y_{k} \in E$ such that $x=y_{1} \cdots y_{k}$. Partition $\{1, \ldots, k\}$ into disjoint, nonempty subsets $J(1), \ldots, J(m)$, where $m \leq n$, such that $y_{r} \in C_{i}$ for all $r \in J(i)$. For each $i$, the set $\left\{y_{r}: r \in J(i)\right\}$ is totally ordered (as a subset of $S$ ) and so has a least element, say $y_{s(i)}$. Then $\prod_{r \in J(i)} y_{r}=y_{s(i)}$, so

$$
x=y_{s(1)} \ldots y_{s(m)} \in\langle E\rangle_{m} \subseteq\langle E\rangle_{n}
$$

and thus $b_{\mathrm{loc}}(E) \leq n$.)

ExAmple 3.11. Let $n \geq 2$. If $S$ has height at most $n$ (that is, each chain in $S$ has cardinality at most $n$ ) then $b(S) \leq n$.

Proof. Let $E \subseteq S$ and $x \in\langle E\rangle$. For some integer $k$ there exists $y_{1}, \ldots, y_{k} \in E$ such that $x=y_{1} \cdots y_{k}$. Clearly $y_{1} \geq y_{1} y_{2} \geq \cdots \geq y_{1} y_{2} \cdots y_{k}$. Let

$$
J=\left\{r \in\{2, \ldots, k\}: y_{1} \cdots y_{r-1} \neq y_{1} \cdots y_{r}\right\}
$$

and enumerate its elements in increasing order as $j_{1}<\cdots<j_{m}$. Since chains in $S$ have cardinality at most $n$, we have $m \leq n+1$, and thus $b_{\text {loc }}(E) \leq n$.)

We need one more technical definition. The following concept is a slight improvement, suggested by the referee, of the author's original condition. However, the author takes the blame for the nonstandard terminology. 
Definition 3.12. Let $(S, \omega)$ be a weighted semilattice. For each $K>0$, let

$$
W_{K}=\{x \in S: \omega(x) \leq K\} .
$$

We say that $(S, \omega)$ is flighty if, for each $K>0$,

$$
\sup \left\{\omega(y): y \in\left\langle W_{K}\right\rangle\right\}<\infty .
$$

This condition is somewhat artificial, and is set up to make the proof of Theorem 3.14 work. Let us first consider some examples.

Example 3.13. (i) If $\sup _{x \in S} \omega(x)<\infty$, then $(S, \omega)$ is flighty.

(ii) If $S$ has finite breadth $n$, then $(S, \omega)$ is flighty for any weight $\omega$; for given $K>0$, we have $\omega(y) \leq K^{n}$ for all $y \in\left\langle W_{K}\right\rangle$.

(iii) Let $(T, \omega)$ be the weighted semilattice constructed in the proof of Theorem 3.4. This example is not flighty: since (if $C$ is the constant used to define the weight in that example) we can for each $n$ find $x_{1}, \ldots, x_{n} \in W_{C}$ such that $\omega\left(x_{1} \cdots x_{n}\right)=C^{n}$.

We can now state the main result of this section. The proof we give incorporates a small simplification suggested by the referee, in line with his or her suggested modification of Definition 3.12.

Theorem 3.14. Let $(S, \omega)$ be a weighted semilattice. If $(S, \omega)$ is flighty, then $\ell_{\omega}^{1}(S)$ is AMNM.

Proof. We will use Corollary 3.7. Fix $\varepsilon>0$, and let

$$
S_{\mathrm{fix}}:=W_{2 / \varepsilon} \equiv\{x \in S: \omega(x) \leq 2 / \varepsilon\},
$$

which we think of as the set where $\omega$ is relatively small.

By our assumption on $(S, \omega)$, there exists some constant $C(\varepsilon)>0$ such that $\omega(y) \leq$ $C(\varepsilon)$ for all $y \in\left\langle S_{\mathrm{fix}}\right\rangle$. Choose $\delta>0$ such that $2 \delta C(\varepsilon) / \varepsilon<1$.

Now suppose that $\psi: S \rightarrow\{0,1\}$ satisfies $\operatorname{def}_{\omega}(\psi) \leq \delta$. Let

$$
E:=\left\{y \in S_{\mathrm{fix}}: \psi(y)=1\right\}
$$

and let

$$
F:=\bigcup_{y \in\langle E\rangle}\{x \in S: x \geq y\} .
$$

Recall that if $E$ is empty then so is $F$; otherwise $F$ is the filter generated by $E$ in $S$.

To motivate the next step, suppose that $\phi: S \rightarrow \mathbb{C}$ is multiplicative and is close in norm to $\psi$. Then (provided $\delta$ is sufficiently small) we must have $\phi=1$ on $E$; and so, as remarked in Lemma 2.8, $\phi$ must be 1 on $F$. Therefore, $\psi$ must also be 1 on $F \cap S_{\text {fix }}$, that is, we must have $E=F \cap S_{\text {fix }}$.

So our next step is to verify that $E=F \cap S_{\text {fix }}$. It suffices to show that $F \cap S$ fix $\subseteq E$, and the proof of this goes via the following claim.

Claim. Let $k \in \mathbb{N}$. Then $\psi(y)=1$ for all $y \in\langle E\rangle_{k}$. 
Proof of Claim. The proof is by induction on $k$. If $k=1$ there is nothing to prove. Suppose that the claim holds for $k=m-1$ where $2 \leq m$, and let $x_{1}, \ldots, x_{m} \in E$. Put $y^{\prime}=x_{1} \cdots x_{m-1}$; then by the inductive hypothesis, the definition of the constant $C(\varepsilon)$, and our choice of $\delta$,

$$
\begin{aligned}
\left|\psi\left(y^{\prime} x_{m}\right)-1\right| & =\left|\psi\left(y^{\prime} x_{m}\right)-\psi\left(y^{\prime}\right) \psi\left(x_{m}\right)\right| \\
& \leq \delta \omega\left(y^{\prime}\right) \omega\left(x_{m}\right) \\
& \leq \delta C(\varepsilon) \omega\left(x_{m}\right) \\
& \leq \delta C(\varepsilon) 2 / \varepsilon<1
\end{aligned}
$$

Since $\psi$ is $0-1$-valued, this forces $\psi\left(y^{\prime} x_{m}\right)=1$, so that the claim holds for $k=m$.

Now let $z \in F \cap S_{\text {fix }}$. Since $z \in F$, there exists $y \in\langle E\rangle$ such that $z y=y$. By our claim, $\psi(y)=1$. Since $z \in S_{\text {fix }}$ and $E \subseteq S_{\text {fix }}$, this implies that

$$
\begin{aligned}
|\psi(z)-1| & =|\psi(y) \psi(z)-\psi(y z)| \\
& \leq \delta \omega(y) \omega(z) \\
& \leq \delta C(\varepsilon) \omega(z) \\
& \leq \delta C(\varepsilon) 2 / \varepsilon<1
\end{aligned}
$$

As before, this forces $\psi(z)=1$, so that $z \in E$.

Thus $F \cap S_{\text {fix }}=E$, as required. We therefore have $S=E \cup\left(S_{\text {fix }} \backslash F\right) \cup\left(S \backslash S_{\text {fix }}\right)$. Now observe that:

- $\quad$ when $x \in E$, we have $\left|\chi_{F}(x)-\psi(x)\right|=0$;

- $\quad$ when $x \in S_{\text {fix }} \backslash F \subseteq S_{\text {fix }} \backslash E$, we have $\chi_{F}(x)=0=\psi(x)$, so that $\left|\chi_{F}(x)-\psi(x)\right|=$ 0

- $\quad$ when $x \in S \backslash S_{\text {fix }}$, we have $\omega(x) \geq 2 / \varepsilon$, so that $\left|\chi_{F}(x)-\psi(x)\right| \leq 2 \leq \varepsilon \omega(x)$.

Putting these cases together, we see that $\chi_{F}$ is multiplicative and satisfies

$$
\sup _{x \in S} \omega(x)^{-1}\left|\chi_{F}(x)-\psi(x)\right| \leq \varepsilon .
$$

In view of Corollary 3.7, this shows that $\ell_{\omega}^{1}(S)$ is AMNM.

As a special case of Theorem 3.14, we get another proof that $\ell^{1}(S)$ is AMNM for every semilattice $S$ (see Example 3.13(i)). More interestingly, we can deduce that if $S$ is a semilattice with either finite width or finite height, then $\ell_{\omega}^{1}(S)$ is AMNM for every submultiplicative weight $\omega$ (see Example 3.13(ii)). In particular, for any weight function $\omega: \mathbb{N} \rightarrow[1, \infty)$, the algebra $B_{\omega}$ from Example 2.5 is AMNM, as it is isomorphic to $\ell_{\omega}^{1}\left(\mathbb{N}_{\min }\right)$.

\section{More general range algebras}

For which Banach algebras $B$ and weighted semilattices $(S, \omega)$ is $\left.\left(\ell_{\omega}^{1}(S), B\right)\right)$ an AMNM pair? Since we do not have a complete answer in the case $B=\mathbb{C}$, we can 
expect only partial results in the more general case. A natural place to start is with those semilattices covered by Theorem 3.14, in particular with $\mathbb{N}_{\text {min }}$. Recall that by this theorem, $\ell_{\omega}^{1}\left(\mathbb{N}_{\text {min }}\right)$ is AMNM for any weight $\omega$. In contrast, we will see shortly that for most choices of nontrivial weight $\omega$ on $\mathbb{N}_{\min }$, the pairs $\left(\ell_{\omega}^{1}\left(\mathbb{N}_{\min }\right), \mathbf{T}_{2}\right)$ and $\left(\ell_{\omega}^{1}\left(\mathbb{N}_{\text {min }}\right), \mathbb{M}_{2}\right)$ fail to be AMNM. Here, $\mathbb{M}_{2}$ is the algebra of $2 \times 2$ complex matrices, and $\mathbf{T}_{2}$ is the subalgebra

$$
\left\{\left(\begin{array}{ll}
a & b \\
0 & a
\end{array}\right): a, b \in \mathbb{C}\right\} \cong \mathbb{C}[x] /\left(x^{2}\right) .
$$

REMARK 4.1. These are not arbitrary test cases: $\mathbb{M}_{2}$ is the smallest semisimple algebra that is noncommutative, while $\mathbf{T}_{2}$ is the smallest commutative, unital algebra that is not semisimple. (In the context of commutative algebra and deformation theory, it is also known as the algebra of dual numbers over $\mathbb{C}$, since it formalizes the notion of an infinitesimal element vanishing to second order.) Additional motivation comes from results of Howey, who showed that $C^{k}[0,1]$ is $\operatorname{AMNM}(k \geq 1)$ [7], while observing - in slightly different notation - that the pair $\left(C^{k}[0,1], \mathbf{T}_{2}\right)$ is not AMNM ([6, Corollary 4.2.5]; see also Remark 4.3 below).

It does not matter which norm we put on $\mathbf{T}_{2}$ or $\mathbb{M}_{2}$. To be definite, we give $\mathbb{M}_{2}$ its natural norm (the $\mathrm{C}^{*}$-algebra norm), but equip $\mathbf{T}_{2}$ with the norm

$$
\left\|\left(\begin{array}{ll}
a & b \\
0 & a
\end{array}\right)\right\|_{\mathbf{T}_{2}}=|a|+|b| .
$$

We now consider AMNM pair problems for $\ell_{\omega}^{1}\left(\mathbb{N}_{\min }\right)$ when the range is $\mathbf{T}_{2}$ or $\mathbb{M}_{2}$. If the weight function is bounded then $\ell_{\omega}^{1}\left(\mathbb{N}_{\min }\right)$ is isomorphic as a Banach algebra to $\ell^{1}\left(\mathbb{N}_{\text {min }}\right)$, and so we may restrict attention to the cases where the weight is either trivial (that is, identically 1) or unbounded.

Theorem 4.2. Let $\omega$ be an unbounded weight function on $\mathbb{N}_{\min }$. Then $\left(\ell_{\omega}^{1}\left(\mathbb{N}_{\min }\right), \mathbf{T}_{2}\right)$ is not an AMNM pair.

Proof. Throughout this proof, let $A$ denote the algebra $\ell_{\omega}^{1}\left(\mathbb{N}_{\min }\right)$. For each $m \in \mathbb{N}$ let $\chi_{m}$ be the character

$$
\chi_{m}(k)= \begin{cases}1 & \text { if } k \geq m, \\ 0 & \text { if } k<m,\end{cases}
$$

and let $\delta_{m}$ be the Dirac point mass at $m$. Set

$$
\theta_{m}=\left(\begin{array}{cc}
\chi_{m} & \omega(m) \delta_{m} \\
0 & \chi_{m}
\end{array}\right): \mathbb{N} \rightarrow \mathbf{T}_{2},
$$

noting that the range of $\theta_{m}$ consists of commuting elements. 
For $1 \leq j \leq k$,

$$
\begin{aligned}
\theta_{m}(j) \theta_{m}(k)-\theta_{m}(j) & =\left(\begin{array}{cc}
0 & \chi_{m}(j) \omega(m) \delta_{m}(k)+\omega(m) \delta_{m}(j)\left(\chi_{m}(k)-1\right) \\
0 & 0
\end{array}\right) \\
& =\left(\begin{array}{cc}
0 & \chi_{m}(j) \omega(m) \delta_{m}(k) \\
0 & 0
\end{array}\right) .
\end{aligned}
$$

Hence by symmetry, for general $j, k \in \mathbb{N}_{\min }$,

$$
\theta_{m}(j) \theta_{m}(k)-\theta_{m}(j \wedge k)=\left(\begin{array}{cc}
0 & \chi_{m}(j \wedge k) \omega(m) \delta_{m}(j \vee k) \\
0 & 0
\end{array}\right)
$$

which vanishes unless $j=k=m$, in which case

$$
\theta_{m}(m) \theta_{m}(m)-\theta_{m}(m)=\left(\begin{array}{cc}
0 & \omega(m) \\
0 & 0
\end{array}\right)
$$

Thus

$$
\operatorname{def}\left(\theta_{m}\right)=\sup _{j, k \in \mathbb{N}} \frac{\|\theta(j) \theta(k)-\theta(j \wedge k)\|}{\omega(j) \omega(k)}=\frac{\omega(m)}{\omega(m) \omega(m)}=\omega(m)^{-1} .
$$

Suppose that $\Phi: \mathbb{N}_{\min } \rightarrow \mathbf{T}_{2}$ is multiplicative. Since $\mathbf{T}_{2}$ has no nontrivial idempotents, this forces $\phi(n)_{12}=0$ for all $n$. Hence

$$
\left\|\Phi(m)-\theta_{m}(m)\right\| \geq\left|\Phi(m)_{12}-\theta_{m}(m)_{12}\right| \geq \omega(m),
$$

so that

$$
\operatorname{dist}_{\mathcal{L}\left(A, \mathbf{T}_{2}\right)}\left(\theta_{m}, \operatorname{Mult}\left(A, \mathbf{T}_{2}\right)\right) \geq \sup _{x \in \mathbb{N}} \frac{\left\|\Phi(x)-\theta_{m}(x)\right\|}{\omega(x)} \geq 1 \quad \text { for all } m .
$$

Since $\lim \inf _{n} \omega(n)^{-1}=0$, it follows that $\left(A, \mathbf{T}_{2}\right)$ is not an AMNM pair.

Remark 4.3. A (bounded) multiplicative function $\Psi$ from a Banach algebra $A$ to $\mathbf{T}_{2}$ is easily seen to be of the form

$$
\Psi(a)=\left(\begin{array}{cc}
\varphi(a) & D(a) \\
0 & \varphi(a)
\end{array}\right)
$$

where $\varphi \in \operatorname{Mult}(A, \mathbb{C})$ and $D: A \rightarrow \mathbb{C}$ is a (bounded) derivation with respect to the bimodule action of $A$ on $\mathbb{C}$ via $\phi$. In the case where $A$ has a dense subspace consisting of commuting idempotents, the only such bounded derivation is 0 ; and the failure of $\left(\ell_{\omega}^{1}\left(\mathbb{N}_{\min }\right), \mathbf{T}_{2}\right)$ to be AMNM can be interpreted as saying that there are 'approximate point derivations' of large norm on $\ell_{\omega}^{1}\left(\mathbb{N}_{\min }\right)$. This perspective (motivated by cohomological questions about the 'Feinstein algebras') was the original approach used to construct the counterexample seen in proving Theorem 4.2. With hindsight, a similar idea can be seen behind Howey's result that $\left(C^{k}[0,1], \mathbf{T}_{2}\right)$ is not an AMNM pair. 
In general, approximately multiplicative maps into an algebra $B$ can be far from multiplicative maps into $B$, yet be close to multiplicative maps into $C$ for some containing algebra $C \supset B$. This is illustrated by the following example, provided by the referee.

Example 4.4 (Referee's example). Let $\chi_{m}$ and $\theta_{m}$ be as in the proof of Theorem 4.2. We saw in that proof that $\operatorname{def}\left(\theta_{m}\right)=\omega(m)^{-1}$ and

$$
\operatorname{dist}_{\mathcal{L}\left(A, \mathbf{T}_{2}\right)}\left(\theta_{m}, \operatorname{Mult}\left(A, \mathbf{T}_{2}\right)\right) \geq 1 \text {. }
$$

Now define $\phi_{m}: \mathbb{N}_{\min } \rightarrow \mathbb{M}_{2}$ by

$$
\phi_{m}=\left(\begin{array}{cc}
\chi_{m} & \omega(m) \delta_{m} \\
0 & \chi_{m+1}
\end{array}\right) .
$$

A case-by-case analysis confirms that $\phi_{m}$ is multiplicative, the key point being that $\phi_{m}(k)=0$ for $k<m$ and $\phi_{m}(k)=I$ for $k>m$. Moreover,

$$
\theta_{m}-\chi_{m}=\left(\begin{array}{cc}
0 & 0 \\
0 & \delta_{m}
\end{array}\right),
$$

so that $\left\|\theta_{m}-\chi_{m}\right\|=\omega(m)^{-1}=\operatorname{def}\left(\theta_{m}\right)$.

One reason why things are trickier when the range algebra is $\mathbb{M}_{2}$ is that the lattice of idempotents is bigger. Nevertheless, we will be able to use the following modest observation: if $V$ is a finite-dimensional vector space, and $P, Q$ are commuting idempotents in $\mathcal{L}(V)$ with the same rank, then $P=Q$. While this observation is easily proved by geometric considerations, we outline an alternative approach: since $P$ and $Q$ are commuting idempotents, $P-Q$ is idempotent; and since the rank of an idempotent equals its trace, it follows that

$$
\operatorname{rank}(P-Q)=\operatorname{Tr}(P-Q)=\operatorname{Tr}(P)-\operatorname{Tr}(Q)=\operatorname{rank}(P)-\operatorname{rank}(Q)=0
$$

so that $P-Q=0$, as required.

REMARK 4.5. We mention this approach since an 'approximate version' will be used in Section 5 when dealing with $2 \times 2$ matrices that are 'approximately idempotent': while the rank of a matrix behaves badly under small-norm perturbations, its trace behaves much better.

Lemma 4.6. (i) Let $a, b \geq 1$ and let $A=\left(\begin{array}{cc}1 & -a \\ 0 & 0\end{array}\right), B=\left(\begin{array}{ll}1 & b \\ 0 & 0\end{array}\right)$. If $P, Q$ are commuting idempotents in $\mathbb{M}_{2}$, then either $\|P-A\| \geq a / 2$ or $\|Q-B\| \geq b / 2$.

(ii) Let $d \geq 1$ and let $C=\left(\begin{array}{ll}1 & d \\ 0 & 0\end{array}\right)$. If $P, Q$ are commuting idempotents in $\mathbb{M}_{2}$, then either $\|P-2 C\| \geq d / 2$ or $\|Q-C\| \geq d / 4$.

Proof. We prove both (i) and (ii) by contradiction. Suppose that $P$ and $Q$ are commuting idempotents that satisfy $\|P-A\|<a / 2$ and $\|Q-B\|<b / 2$. Since $\|A\| \geq a$ 
and $\|A-I\| \geq a, P \notin\{0, I\}$; similarly, $Q \notin\{0, I\}$. Hence $P$ and $Q$ both have rank one. This forces $P=Q$ (see the observation made before Remark 4.5), and so

$$
0<a+b=\|A-B\| \leq\|A-P\|+\|Q-B\|<(a+b) / 2
$$

which is a contradiction. Thus (i) is proved.

Similarly, suppose that $P$ and $Q$ are commuting idempotents that satisfy $\|P-2 C\|<$ $d / 2$ and $\|Q-C\|<d / 4$. Since $\|C\| \geq d$ and $\|C-I\| \geq d, Q \notin\{0, I\}$; similarly, $P \notin\{0, I\}$. Thus $P$ and $Q$ both have rank one, which as before forces $P=Q$. But then

$$
0<d=\|C\| \leq\|2 C-P\|+\|Q-C\|<\frac{d}{2}+\frac{d}{4}
$$

which is a contradiction. Thus (ii) is proved.

We can now present our main result for $\ell_{\omega}^{1}\left(\mathbb{N}_{\min }\right)$ and $\mathbb{M}_{2}$, which shows that for many unbounded weights we do not get an AMNM pair. The author is grateful to the referee for spotting errors in an earlier version, which mistakenly claimed that the AMNM property failed for all unbounded weights; see also the discussion following Remark 4.8.

THEOREM 4.7. Let $\omega$ be a weight function on $\mathbb{N}_{\min }$ that satisfies

$$
\sup _{n} \min (\omega(n), \omega(n+1))=+\infty \text {. }
$$

Then $\left(\ell_{\omega}^{1}\left(\mathbb{N}_{\text {min }}\right), \mathbb{M}_{2}\right)$ is not an AMNM pair.

Proof. Let $\delta>0$. We will construct an $\omega$-bounded function $\theta: \mathbb{N}_{\min } \rightarrow \mathbb{M}_{2}$ which has norm at most 2 (with respect to the $\ell_{\omega}^{1}$-norm) and satisfies $\operatorname{def}_{\omega}(\theta) \leq \delta$, yet also satisfies $\|\theta-\phi\|_{\infty, \omega^{-1}} \geq 1 / 2$ for every multiplicative function $\phi: \mathbb{N}_{\min } \rightarrow \mathbb{M}_{2}$.

By our assumption on the weight, there exists $n \in \mathbb{N}$ such that $\min (\omega(n), \omega(n+1)) \geq$ $2 / \delta$. We define

$$
\begin{gathered}
\theta(j)=0 \quad \text { for all } j \leq n-1, \\
\theta(n)=\left(\begin{array}{cc}
1 & -\omega(n) \\
0 & 0
\end{array}\right), \\
\theta(n+1)=\left(\begin{array}{cc}
1 & \omega(n+1) \\
0 & 0
\end{array}\right), \\
\theta(k)=I \quad \text { for all } k \geq n+2 .
\end{gathered}
$$

Clearly $\|\theta\|_{\infty . \omega^{-1}} \leq 2$. We claim that $\theta$ is $\delta$-multiplicative. Clearly $\theta(j) \theta(k)=\theta(j)=$ $\theta(k) \theta(j)$ whenever $j \leq n-1$ or $k \geq n+2$. We also have $\theta(n)^{2}=\theta(n), \theta(n+1)^{2}=$ $\theta(n+1), \theta(n+1) \theta(n)=\theta(n)$, and

$$
\theta(n) \theta(n+1)-\theta(n)=\theta(n+1)-\theta(n)=\left(\begin{array}{cc}
0 & \omega(n+1)+\omega(n) \\
0 & 0
\end{array}\right) .
$$


Therefore

$$
\operatorname{def}_{\omega}(\theta)=\frac{\|\theta(n) \theta(n+1)-\theta(n)\|}{\omega(n) \omega(n+1)}=\frac{\omega(n+1)+\omega(n)}{\omega(n) \omega(n+1)}=\frac{1}{\omega(n)}+\frac{1}{\omega(n+1)} \leq \delta,
$$

as claimed.

Finally, let $\phi: \mathbb{N}_{\min } \rightarrow \mathbb{M}_{2}$ be multiplicative. Then $\phi(n)$ and $\phi(n+1)$ are commuting idempotents, so by Lemma 4.6(i),

$$
\text { either }\|\theta(n)-\phi(n)\| \geq \omega(n) / 2 \text { or }\|\theta(n+1)-\phi(n+1)\| \geq \omega(n+1) / 2 \text {. }
$$

Therefore $\|\theta-\phi\|_{\infty, \omega^{-1}} \geq 1 / 2$.

REMARK 4.8. If $\omega$ is any weight on $\mathbb{N}_{\min }$ with $\lim \inf _{n} \omega(n)<\infty$, then $\ell_{\omega}^{1}\left(\mathbb{N}_{\min }\right)$ is known to be approximately amenable (in fact, it is boundedly approximately contractible, by combining the discussion in Example 2.5 with [4, Corollary 4.5]). Thus, Theorems 4.2 and 4.7 show we can have approximately amenable Banach algebras $A$ and finite-dimensional Banach algebras $B$ such that $(A, B)$ is not an AMNM pair, in contrast with what happens when $A$ is amenable [11, Theorem 3.1].

Theorem 4.7 implies a necessary condition on the weight $\omega$ for the pair $\left(\ell_{\omega}^{1}\left(\mathbb{N}_{\min }\right), \mathbb{M}_{2}\right)$ to be $\mathrm{AMNM}$, and one naturally wonders if this necessary condition is sufficient. Put more explicitly: if $\omega: \mathbb{N}_{\min } \rightarrow[1, \infty)$ is a weight function satisfying $\sup _{n} \min (\omega(n), \omega(n+1))<\infty$, is $\left(\ell_{\omega}^{1}\left(\mathbb{N}_{\min }\right), \mathbb{M}_{2}\right)$ always an AMNM pair?

It turns out that the answer to this question is positive. This was discovered after the main work of the present paper, and the current proof is relatively long and unenlightening, while relying on Theorem 5.1. Details will therefore be given in forthcoming work, which treats AMNM problems for more general range algebras. The main idea is similar to that in the proof of Theorem 3.14: given $\delta>0$, one partitions $\mathbb{N}_{\text {min }}$ into a set where the weight is 'large' and one where it is 'small'; then given a $\delta$-multiplicative function $\theta: \ell_{\omega}^{1}\left(\mathbb{N}_{\text {min }}\right) \rightarrow \mathbb{M}_{2}$, one applies an AMNM result for the unweighted case to constrain the values of $\theta$ on the set where the weight is small, and then adjusts $\theta$ on the set where the weight is large.

For the sake of completeness, we include the following result, which should be contrasted with Theorem 5.1.

Theorem 4.9. Let $\omega$ be an unbounded weight on $\mathbb{N}_{\text {min }}$. Then $\left(\ell_{\omega}^{1}\left(\mathbb{N}_{\min }\right), \mathbb{M}_{2}\right)$ is not a uniformly AMNM pair.

Proof. Since uniformly AMNM pairs are a fortiori AMNM, it suffices by Theorem 4.7 to consider the case where $\omega$ is unbounded and $\sup _{n} \min (\omega(n), \omega(n+1))<\infty$.

Our argument is very similar to the proof of Theorem 4.7. Let $C=\sup _{n} \min (\omega(n)$, $\omega(n+1))$, and let $\delta>0$. We will construct an $\omega$-bounded function $\theta: \mathbb{N}_{\min } \rightarrow \mathbb{M}_{2}$ which satisfies $\operatorname{def}_{\omega}(\theta) \leq \delta$, yet also satisfies $\|\theta-\phi\|_{\infty, \omega^{-1}} \geq 1 / 2$ for every multiplicative function $\phi: \mathbb{N}_{\min } \rightarrow \mathbb{M}_{2}$. 
The hypotheses on $\omega$ ensure that there exists some $n \in \mathbb{N}$ such that $\omega(n) \geq$ $\max \left(6 \delta^{-1}, 2 C\right)$ and $\omega(n+1) \leq C$. Define $\theta: \mathbb{N}_{\min } \rightarrow \mathbb{M}_{2}$ by setting $\theta(j)=0$ for all $1 \leq j \leq n-1, \theta(k)=I$ for all $k \geq n+2$, and

$$
\theta(n+1):=\left(\begin{array}{cc}
1 & \omega(n) \\
0 & 0
\end{array}\right), \quad \theta(n)=2 \theta(n+1)=\left(\begin{array}{cc}
2 & 2 \omega(n) \\
0 & 0
\end{array}\right) .
$$

Then $\theta$ is an $\omega$-bounded map $\mathbb{N}_{\min } \rightarrow \mathbb{M}_{2}$, with

$$
\begin{aligned}
\|\theta\|_{\infty, \omega^{-1}} & =\max \left(\frac{\|\theta(n)\|}{\omega(n)}, \frac{\|\theta(n+1)\|}{\omega(n+1)}\right) \\
& \leq \max \left(\frac{2+2 \omega(n)}{\omega(n)}, \frac{1+\omega(n)}{\omega(n+1)}\right) \leq \frac{2 \omega(n)}{\omega(n+1)} .
\end{aligned}
$$

Clearly $\theta(j) \theta(k)=\theta(j)=\theta(k) \theta(j)$ whenever $1 \leq j \leq n-1$ or $n+2 \leq k$. Furthermore, $\theta(n+1)^{2}=\theta(n+1)$ and $\theta(n) \theta(n+1)=\theta(n)=\theta(n) \theta(n+1)$, while $\theta(n)^{2}-\theta(n)=$ $3 \theta(n+1)$. Therefore

$$
\operatorname{def}_{\omega}(\theta)=\frac{\left\|\theta(n)^{2}-\theta(n)\right\|}{\omega(n)^{2}}=\frac{3\|\theta(n+1)\|}{\omega(n)^{2}} \leq \frac{6}{\omega(n)} \leq \delta .
$$

Now let $\phi: \mathbb{N}_{\min } \rightarrow \mathbb{M}_{2}$ be a multiplicative function. Then $\phi(n)$ and $\phi(n+1)$ are commuting idempotents, so by taking $C=\theta(n+1)$ in Lemma 4.6(ii),

$$
\text { either }\|\theta(n)-\phi(n)\| \geq \omega(n) / 2 \text { or }\|\theta(n+1)-\phi(n+1)\| \geq \omega(n) / 4 \geq \omega(n+1) / 2 .
$$

Therefore $\|\theta-\phi\|_{\infty, \omega^{-1}} \geq 1 / 2$.

Theorems 4.2 and 4.7 suggest that if we seek positive results, we are better off considering the unweighted convolution algebras of semilattices. This is corroborated by the final result of this section.

THeOREM 4.10. Let $S$ be a semilattice. Then $\left(\ell^{1}(S), \mathbf{T}_{2}\right)$ is a uniformly AMNM pair.

Proof. Let $\theta: S \rightarrow \mathbf{T}_{2}$ be a function satisfying $\operatorname{def}(\theta)<1 / 5$, and let $a, b: S \rightarrow \mathbb{C}$ be the functions defined by

$$
\theta(e)=\left(\begin{array}{cc}
a(e) & b(e) \\
0 & a(e)
\end{array}\right), \quad e \in S .
$$

Since

$$
\left(\begin{array}{cc}
a(e) & b(e) \\
0 & a(e)
\end{array}\right)\left(\begin{array}{cc}
a(f) & b(f) \\
0 & a(f)
\end{array}\right)=\left(\begin{array}{cc}
a(e) a(f) & a(e) b(f)+b(e) a(f) \\
0 & a(e) a(f)
\end{array}\right),
$$

we see that $\operatorname{def}(a) \leq \operatorname{def}(\theta)<1 / 5$. By Proposition 3.3, there exists a multiplicative function $\chi: S \rightarrow \mathbb{C}$ such that

$$
|a(e)-\chi(e)|<\frac{7}{5}\left|a(e)^{2}-a(e)\right|<\frac{7}{5} \operatorname{def}(\theta)<7 / 25, \quad \text { for all } e \in S .
$$


Note that

$$
\begin{aligned}
\operatorname{def}(\theta) & \geq \sup _{e \in S}\left\|\theta(e)^{2}-\theta(e)\right\| \\
& =\sup _{e \in S}\left\|\left(\begin{array}{cc}
a(e)^{2}-a(e) & (2 a(e)-1) b(e) \\
0 & a(e)^{2}-a(e)
\end{array}\right)\right\| \\
& =\sup _{e \in S}\left|a(e)^{2}-a(e)\right|+|2 a(e)-1||b(e)| .
\end{aligned}
$$

Let $e \in S$. Since $\left|a(e)^{2}-a(e)\right| \leq \operatorname{def}(a) \leq \operatorname{def}(\theta)<1 / 5$, applying Lemma 3.2 and using the estimate (3.2) yields

$$
\min (|a(e)|,|1-a(e)|)<\rho\left(\frac{1}{5}\right) \operatorname{def}(a)<\frac{7}{25} ;
$$

therefore $a(e) \in \mathbb{D}_{0}(7 / 25) \cup \mathbb{D}_{1}(7 / 25)$, so that

$$
\left|\frac{1}{2}-a(e)\right| \geq \frac{1}{2}-\frac{7}{25}=\frac{11}{50} .
$$

Hence

$$
\begin{aligned}
\|\theta(e)-\chi(e) I\| & =|a(e)-\chi(e)|+|b(e)| \\
& \leq \frac{7}{5}\left|a(e)^{2}-a(e)\right|+\frac{25}{11}|(2 a(e)-1) b(e)| \leq \frac{25}{11} \operatorname{def}(\theta),
\end{aligned}
$$

as required.

It is now natural to ask if $\left(\ell^{1}(S), \mathbb{M}_{2}\right)$ is an AMNM pair. The answer turns out to be yes - in fact, it is always a uniformly AMNM pair - but the proof is considerably harder, and occupies all of the next section.

\section{5. $\left(\ell^{1}(S), \mathbb{M}_{2}\right)$ is AMNM for any semilattice $S$}

For reasons of technical convenience, we shall work mostly with the HilbertSchmidt norm on $\mathbb{M}_{2}$, defined by $\|A\|_{\mathrm{HS}}^{2}=\operatorname{Tr}\left(A^{*} A\right)^{1 / 2}$. It might be conceptually clearer to use the operator norm throughout, but this seems to yield worse constants in later inequalities, which are obtained by bootstrapping up the earlier ones.

Notation. If $A \in \mathbb{M}_{2}$ and $\varepsilon>0$ let

$$
\overline{\mathbb{B}}_{A}^{\mathrm{HS}}(\varepsilon)=\left\{B \in \mathbb{M}_{2}:\|A-B\|_{\mathrm{HS}} \leq \varepsilon\right\}
$$

(the closed ball of radius $\varepsilon$ centred on $A$, in the Hilbert-Schmidt norm).

Theorem 5.1. Let $\delta<0.03$, and let $\theta: S \rightarrow \mathbb{M}_{2}$ be a function satisfying

$$
\sup _{e \cdot f \in S}\|\theta(e) \theta(f)-\theta(e f)\|_{\mathrm{HS}} \leq \delta .
$$

Then there exists a (bounded) multiplicative function $\phi: S \rightarrow \mathbb{M}_{2}$ such that

$$
\sup _{x \in S}\|\theta(x)-\phi(x)\|_{\mathrm{HS}} \leq 12 \delta .
$$

In particular, $\left(\ell^{1}(S), \mathbb{M}_{2}\right)$ is a uniformly AMNM pair. 
Note that we do not assume in (5.1) that $\sup _{e \in S}\|\theta(e)\|_{\mathrm{HS}}<\infty$, but this will emerge during the proof (see Proposition 5.4).

5.1. Motivating the proof of Theorem 5.1. Let $S$ be a semilattice. Our proof that $\left(\ell^{1}(S), \mathbb{C}\right)$ is a uniformly AMNM pair can be broken down into three steps.

(1) Show that there is a constant $c$, such that whenever $\theta: S \rightarrow \mathbb{C}$ is $\delta$-multiplicative, $\theta(S) \subseteq \overline{\mathbb{D}}_{0}(c \delta) \cup \overline{\mathbb{D}}_{1}(c \delta)$. (In effect, this step 'approximately discretizes' the problem.)

(2) Put $S_{k}:=\theta^{-1}\left(\overline{\mathbb{D}}_{k}(c \delta)\right)$ for $k=0,1$, so that $S$ is partitioned as $S_{1} \cup S_{0}$. Show that $S_{1} \cdot S_{1} \subseteq S_{1}, S_{1} \cdot S_{0} \subseteq S_{0}$, and $S_{0} \cdot S_{0} \subseteq S_{0}$. (Although we did not do these calculations explicitly, they are implicit in the process of checking that $S_{1}$ is a filter in $S$.)

(3) Define $\phi: S \rightarrow\{0,1\}$ by $\phi=1$ on $S_{1}$ and $\phi=0$ on $S_{0}$. By the first step, $\|\phi-\theta\|_{\infty} \leq c \delta$, and by the second step, $\phi$ is multiplicative.

The strategy we shall adopt is to mimic each of these steps, but now allow our maps to take values in $\mathbb{M}_{2}$ rather than $\mathbb{C}$. As a first step, we need some characterization of multiplicative functions $\phi: S \rightarrow \mathbb{M}_{2}$, which reduces down to the problem of describing the possible semilattices inside the multiplicative semigroup $\mathbb{M}_{2}$. This is not too hard, once we make the following observation: if $P \in \mathbb{M}_{2}$ is a rank-one idempotent, then the only idempotents which commute with $P$ are $I, P, I-P$ and 0 . (We will see later that there is an 'approximate version' of this.)

Secondly, observe that if $\theta$ is - as claimed - a perturbation of a multiplicative function $\phi$, then by the previous remarks $\theta(S)$ should be contained in a small-ball neighbourhood of $\phi(S)$, which in turn is contained in a set of at most four commuting idempotents. To prove Theorem 5.1, we reverse this line of reasoning, and identify a commuting set $L$ of idempotents in $\mathbb{M}_{2}$, a small-ball neighbourhood of which will contain $\theta(S)$. (See Proposition 5.4 for the details.) Then, since the elements of $L$ are well separated, there is only one realistic candidate for the map $\phi$ : namely, it should send a given $x \in S$ to the element of $L$ nearest to $\theta(x)$. This map $\phi$ will clearly be close in norm to $\theta$, so all that will remain is to check that $\phi$ is multiplicative: this can be done through a case-by-case analysis, although some work is needed since we do not assume that $\sup _{e \in S}\|\theta(e)\|_{\mathrm{HS}}<\infty$.

To identify the set $L$, we make heavy use of a small but technical result, based on the following idea: the trace of an approximately idempotent $2 \times 2$ matrix must be close to an integer, which then equals the rank of any nearby idempotent. This will be made precise in the next lemma.

5.2. A key technical lemma. The following lemma is our basic tool for working with approximately idempotent elements of $\mathbb{M}_{2}$. It is here that the Hilbert-Schmidt norm seems to be convenient.

Lemma 5.2 (Key estimates). (a) Let $\rho(0)=1$ and

$$
\rho(t)=\frac{1}{2 t}(1-\sqrt{1-4 t}), \quad 0<t \leq 1 / 4,
$$


and let

$$
\kappa(t)=(1-\rho(t) t \sqrt{2})^{-1}, \quad 0 \leq t \leq 1 / 4 .
$$

Then $\rho$ and $\kappa$ are increasing functions, with $\rho(t) \leq \kappa(t)$ for all $t \in[0,1 / 4]$. Moreover,

$$
\rho\left(\frac{n}{(n+1)^{2}}\right)=\frac{n+1}{n}
$$

and

$$
\kappa\left(\frac{n}{(n+1)^{2}}\right)=\left(1-\frac{\sqrt{2}}{n+1}\right)^{-1}<\left(1-\frac{10}{7(n+1)}\right)^{-1} .
$$

(b) Let $A \in \mathbb{M}_{2}$ satisfy $\left\|A-A^{2}\right\|_{\mathrm{HS}} \leq \varepsilon<2 / 9$. Then

$$
\|2 A-I\|_{\mathrm{HS}} \geq\left(2-6\left\|A-A^{2}\right\|_{\mathrm{HS}}\right)^{1 / 2}
$$

and

$$
\operatorname{Tr}(A) \in \bigcup_{j \in\{0,1,2\}} \overline{\mathbb{D}}_{j}(\sqrt{2} \rho(\varepsilon) \varepsilon) \subseteq \bigcup_{j \in\{0,1,2\}} \overline{\mathbb{D}}_{j}(10 / 21)
$$

\section{Moreover:}

- $\quad$ if $|\operatorname{Tr}(A)-2|<1 / 2$, then $\|I-A\|_{\mathrm{HS}} \leq \kappa(\varepsilon) \varepsilon$;

- $\quad$ if $|\operatorname{Tr}(A)|<1 / 2$, then $\|A\|_{\mathrm{HS}} \leq \kappa(\varepsilon) \varepsilon$;

- if $|\operatorname{Tr}(A)-1|<1 / 2$ then there exists a rank-one idempotent $P \in \mathbb{M}_{2}$ satisfying $\|I-P\|_{\mathrm{HS}} \leq \rho(\varepsilon) \varepsilon$.

Proof of (a). The formulas (5.2) and (5.3) follow from the definitions of the functions $\rho$ and $\kappa$ by direct calculation. Moreover, we have already seen (see the remarks after the formula (3.1)) that $\rho$ is increasing on $[0,1 / 4]$, and therefore $\kappa$ is also increasing on $[0,1 / 4]$.

It only remains to prove that $\rho(t) \leq \kappa(t)$ for all $t \in[0,1 / 4]$. Since $1=\kappa(0)=\rho(0)<$ $\rho(t)$ for all $0<t \leq 1 / 4$, it suffices to show that $\kappa(t)^{-1}<\rho(t)^{-1}$ for all such $t$. To do this, observe that

$$
\begin{aligned}
\kappa(t)^{-1}+\sqrt{2}-1 & =\sqrt{2}-\sqrt{2} \rho(t) t \\
& =\sqrt{2}\left(1-\frac{1}{2}(1-\sqrt{1-4 t})\right) \\
& =\sqrt{2}\left(\frac{1}{2}+\frac{1}{2} \sqrt{1-4 t}\right),
\end{aligned}
$$

while

$$
(1+\sqrt{1-4 t}) \rho(t)=\frac{1}{2 t}(1-(1-4 t))=2 ;
$$

combining these two identities yields

$$
\kappa(t)^{-1}+\sqrt{2}-1=\sqrt{2} \rho(t)^{-1},
$$

so that

$$
\kappa(t)^{-1}-\rho(t)^{-1}=(\sqrt{2}-1) \rho(t)^{-1}-(\sqrt{2}-1)<0
$$

as required. 
Proof of $(b)$. By conjugating with an appropriate unitary matrix, we may assume without loss of generality that $A$ is upper triangular, say $A=\left(\begin{array}{ll}a & b \\ 0 & d\end{array}\right)$. Then

$$
A-A^{2}=\left(\begin{array}{cc}
a-a^{2} & b(1-a-d) \\
0 & d-d^{2}
\end{array}\right)
$$

so that

$$
\left|a-a^{2}\right|^{2}+|b(1-a-d)|^{2}+\left|d-d^{2}\right|^{2} \leq\left\|A-A^{2}\right\|_{\mathrm{HS}}^{2} \leq \varepsilon^{2} .
$$

Therefore,

$$
\begin{aligned}
\|2 A-I\|_{\mathrm{HS}}^{2} & \geq|2 a-1|^{2}+|2 d-1|^{2} \\
& \geq 2-4\left|a-a^{2}\right|-4\left|d-d^{2}\right| \\
& \geq 2-4 \sqrt{2}\left(\left|a-a^{2}\right|^{2}+\left|d-d^{2}\right|^{2}\right)^{1 / 2} \\
& \geq 2-6\left(\left|a-a^{2}\right|^{2}+\left|d-d^{2}\right|^{2}\right)^{1 / 2} \\
& \geq 2-6|| A-A^{2} \|_{\mathrm{HS}},
\end{aligned}
$$

and we have proved (5.4).

It also follows from (5.6), by using Lemma 3.2, that

$$
\operatorname{dist}_{\mathbb{C}}(a,\{0,1\}) \leq \rho(\varepsilon)\left|a-a^{2}\right| \leq \rho\left(\frac{2}{9}\right) \frac{2}{9}=\frac{1}{3}
$$

and

$$
\operatorname{dist}_{\mathbb{C}}(d,\{0,1\}) \leq \rho(\varepsilon)\left|d-d^{2}\right| \leq \rho\left(\frac{2}{9}\right) \frac{2}{9}=\frac{1}{3} .
$$

Define the function $N: \overline{\mathbb{D}}_{0}(1 / 3) \cup \overline{\mathbb{D}}_{1}(1 / 3) \rightarrow\{0,1\}$ to take the value $i$ on $\overline{\mathbb{D}}_{i}(1 / 3)$ for $i=0,1$. By Cauchy-Schwarz,

$$
\begin{aligned}
|a-N(a)|+|d-N(d)| & \leq \rho(\varepsilon)\left(\left|a-a^{2}\right|+\left|d-d^{2}\right|\right) \\
& \leq \rho(\varepsilon) \sqrt{2}\left(\left|a-a^{2}\right|^{2}+\left|d-d^{2}\right|^{2}\right)^{1 / 2} \leq \rho(\varepsilon) \sqrt{2} \varepsilon ;
\end{aligned}
$$

and since $\rho$ is an increasing function,

$$
\rho(\varepsilon) \sqrt{2} \varepsilon \leq \frac{10}{7} \rho(\varepsilon) \varepsilon \leq \frac{10}{7} \rho\left(\frac{2}{9}\right) \frac{2}{9}=\frac{10}{21} .
$$

Observe that if $r \in \mathbb{Z}$ satisfies $|\operatorname{Tr}(A)-r| \leq 1 / 2$, then

$$
|r-N(a)-N(d)|<\frac{1}{2}+\frac{10}{21}<1,
$$

forcing $r=N(a)+N(d)$. So $r \in\{0,1,2\}$, and we always have

$$
|\operatorname{Tr}(A)-r|=|a+d-N(a)-N(d)|<\rho(\varepsilon) \sqrt{2} \varepsilon \leq \frac{10}{21},
$$

giving us the inclusions in (5.5). 
Finally, we show that $A$ is always $\|\cdot\|_{\text {HS }}$-close to an idempotent of the appropriate rank. We first address the cases where exactly one of $N(a)$ and $N(d)$ is equal to 1 , with the other being equal to 0 . In both of these cases we define

$$
P=\left(\begin{array}{cc}
N(a) & b \\
0 & N(d)
\end{array}\right) \text {. }
$$

A short calculation shows that $P=P^{2}$. By construction,

$$
\begin{aligned}
\|A-P\|_{\mathrm{HS}} & =\left(|a-N(a)|^{2}+|d-N(d)|^{2}\right)^{1 / 2} \\
& \leq \rho(\varepsilon)\left(\left|a-a^{2}\right|^{2}+\left|d-d^{2}\right|^{2}\right)^{1 / 2} \leq \rho(\varepsilon)\left\|A-A^{2}\right\|_{\mathrm{HS}} .
\end{aligned}
$$

Secondly, we address the cases where $\operatorname{Tr}(A) \in \mathbb{D}_{0}(1 / 2) \cup \mathbb{D}_{2}(1 / 2)$. If $|\operatorname{Tr}(A)-2|<$ $1 / 2$ we must have $N(a)=N(d)=1$ (so $D=I$ ) and then, using $(*)$,

$$
|1-a-d| \geq 1-|a+d-2|=1-|a+d-N(a)-N(d)| .
$$

On the other hand, if $|\operatorname{Tr}(A)|<1 / 2$, we must have $N(a)=N(d)=0$ (so $D=0$ ) and then

$$
|1-a-d| \geq 1-|a+d|=1-|a+d-N(a)-N(d)| .
$$

Thus, in both of these cases, by using (*) we obtain the inequality

$$
(|1-a-d|)^{-1} \leq(1-|a+d-N(a)-N(d)|)^{-1} \leq(1-\rho(\varepsilon) \varepsilon \sqrt{2})^{-1}=\kappa(\varepsilon) .
$$

Put

$$
D=\left(\begin{array}{cc}
N(a) & 0 \\
0 & N(d)
\end{array}\right) ;
$$

this matrix equals either $I$ or 0 , depending on whether $\operatorname{Tr}(A)$ is close to 0 or close to 2 . It follows from $(* *)$ that

$$
\begin{aligned}
\|A-D\|_{\mathrm{HS}}^{2} & =|a-N(a)|^{2}+|d-N(d)|^{2}+|b|^{2} \\
& \leq \rho(\varepsilon)^{2}\left|a-a^{2}\right|^{2}+\rho(\varepsilon)^{2}\left|d-d^{2}\right|^{2}+\kappa(\varepsilon)^{2}|b(1-a-d)|^{2} \\
& \leq \kappa(\varepsilon)^{2}\left(\left|a-a^{2}\right|^{2}+\left|d-d^{2}\right|^{2}+|b(1-a-d)|^{2}\right),
\end{aligned}
$$

where we use the result from part (a) that $\rho(\varepsilon) \leq \kappa(\varepsilon)$. Taking square roots gives $\|A-D\|_{\mathrm{HS}} \leq \kappa(\varepsilon)\left\|A-A^{2}\right\|_{\mathrm{HS}} \leq \kappa(\varepsilon) \varepsilon$, and the proof of our lemma is complete.

5.3. Proof of Theorem 5.1. Our argument is broken into smaller results. For the reader's convenience, the interdependencies are indicated in Figure 1. To save needless repetition, we will assume for the rest of this section that $0 \leq \delta<0.03$. This implies from the outset (by Lemma 5.2(a)) that

$$
\rho(\delta) \leq \kappa(\delta)<\kappa\left(\frac{29}{30^{2}}\right)<\left(1-\frac{1}{21}\right)^{-1}=1.05 .
$$

Let $\theta: S \rightarrow \mathbb{M}_{2}$ satisfy condition (5.1). To simplify formulas, we shall use the following abbreviations: $\widetilde{e}$ stands for $\theta(e), \widetilde{f}$ for $\theta(f), \widetilde{e f}$ for $\theta(e f)$, and so forth. 


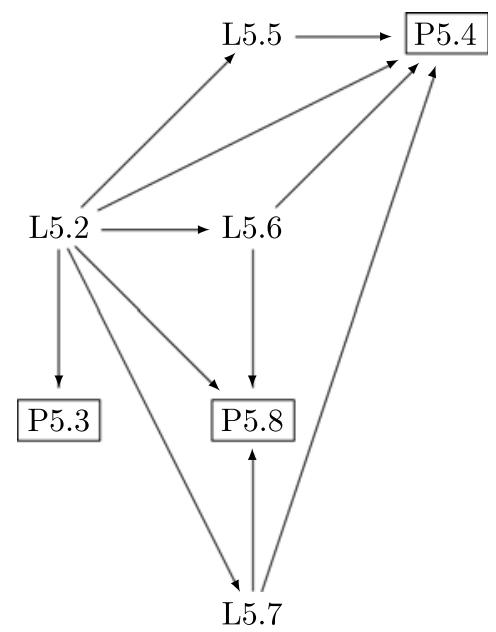

Figure 1. Dependencies between results in this section $(\mathrm{P}=$ Proposition; $\mathrm{L}=\mathrm{Lemma})$.

We start by taking $e=f$ in condition (5.1) and using Lemma 5.2 with $\varepsilon=\delta$. This gives

$$
\operatorname{Tr} \theta(S) \subseteq \bigcup_{j \in\{0,1,2\}} \overline{\mathbb{D}}_{j}(\rho(\delta) \sqrt{2} \delta) \subseteq \bigcup_{j \in\{0,1,2\}} \mathbb{D}_{j}(0.05),
$$

with the second inclusion following from the upper bound

$$
\rho(\delta) \sqrt{2} \delta<1.05 \times \frac{10}{7} \times 0.03<0.05
$$

For $k=0,1,2$, define

$$
S_{k}:=(\operatorname{Tr} \circ \theta)^{-1}\left(\mathbb{D}_{k}(0.95)\right)=(\operatorname{Tr} \circ \theta)^{-1}\left(\overline{\mathbb{D}}_{k}(\rho(\delta) \sqrt{2} \delta)\right) .
$$

Then, by Lemma 5.2(b), $\theta\left(S_{2}\right) \subseteq \overline{\mathbb{B}}_{I}^{\mathrm{HS}}(\kappa(\delta) \delta)$ and $\theta\left(S_{0}\right) \subseteq \overline{\mathbb{B}}_{0}^{\mathrm{HS}}(\kappa(\delta) \delta)$.

Define $\phi: S_{2} \sqcup S_{0} \rightarrow \mathbb{M}_{2}$ by $\phi\left(S_{0}\right)=\{0\}$ and $\phi\left(S_{2}\right)=\{I\}$. By our initial remarks concerning $\kappa(\delta)$,

$$
\sup _{x \in S_{2} \sqcup S_{0}}\|\widetilde{x}-\phi(x)\|_{\mathrm{HS}} \leq \kappa(\delta) \delta \leq 1.05 \delta
$$

Proposition 5.3 (Some easy properties). Let e, $f \in S$.

(i) If $f \in S_{2}$ then $\left\|\widetilde{f}^{-1}\right\|_{\mathrm{HS}}<1.5$. If $e \in S_{0}$ then $\left\|(I-\widetilde{e})^{-1}\right\|_{\mathrm{HS}}<1.5$.

(ii) If $e, f \in S_{2}$ then ef $\in S_{2}$.

(iii) If $e \in S_{0}$ and $f \in S$ then ef $\in S_{0}$.

In particular, $S_{2} \sqcup S_{0}$ is a subsemigroup of $S$, and $\phi: S_{2} \sqcup S_{0} \rightarrow \mathbb{M}_{2}$ is multiplicative. 
Proof. Suppose that $f \in S_{2}$. As remarked above, $\|\widetilde{f}-I\|_{\mathrm{HS}} \leq \kappa(\delta) \delta \leq 1.05 \delta<0.04$. Therefore, using submultiplicativity of the Hilbert-Schmidt norm,

$$
\begin{aligned}
\left\|\widetilde{f}^{-1}\right\|_{\mathrm{HS}} & =\left\|\sum_{n=0}^{\infty}(I-\widetilde{f})^{n}\right\|_{\mathrm{HS}} \\
& \leq\|I\|_{\mathrm{HS}}+\sum_{n=1}^{\infty}\|I-\widetilde{f}\|_{\mathrm{HS}}^{n} \\
& \leq \sqrt{2}-1+\frac{1}{1-\|I-\widetilde{f}\|_{\mathrm{HS}}}<\frac{3}{7}+\frac{1}{0.96}<1.5 .
\end{aligned}
$$

A similar argument shows that when $e \in S_{0}$, we have $\left\|(I-\widetilde{e})^{-1}\right\|_{\mathrm{HS}}<1.5$. This proves part (i).

Now, for any $e, f \in S$,

$$
\|(\widetilde{e f}-\widetilde{e}) \widetilde{f}\|_{\mathrm{HS}} \leq\|\widetilde{e f} \widetilde{f}-\widetilde{e f}\|_{\mathrm{HS}}+\|\widetilde{e f}-\widetilde{e} \widetilde{f}\|_{\mathrm{HS}} \leq 2 \delta .
$$

If $e, f \in S_{2}$, we may combine this upper bound with part (i) to obtain (via CauchySchwarz)

$$
\begin{aligned}
|\operatorname{Tr}(\widetilde{e f})-2| & \leq|\operatorname{Tr}(\widetilde{e})-2|+|\operatorname{Tr}(\widetilde{e f}-\widetilde{e})| \\
& \leq 0.05+\|(\widetilde{e f}-\widetilde{e}) \widetilde{f}\|_{\mathrm{HS}}\left\|\widetilde{f}^{-1}\right\|_{\mathrm{HS}} \leq 0.05+3 \delta \ll 0.95,
\end{aligned}
$$

which implies that $e f \in S_{2}$, by the definition in (5.8). This proves (ii).

Observe that $\|\widetilde{e} \widetilde{e f}-\widetilde{e f}\|_{\mathrm{HS}} \leq \delta$. If $e \in S_{0}$ then, by part (i), $\left\|(\widetilde{e}-I)^{-1}\right\|_{\mathrm{HS}}<1.5$, and so by Cauchy-Schwarz,

$$
|\operatorname{Tr} \widetilde{e f}| \leq\left\|(\widetilde{e}-I)^{-1}\right\|_{\mathrm{HS}}\|(\widetilde{e}-I) \widetilde{e f}\|_{\mathrm{HS}} \leq 1.5 \delta \ll 0.5 .
$$

Hence $e f \in S_{0}$, by (5.8), proving (iii). The final statement of the proposition now follows easily from (ii) and (iii).

Combining (5.9) and Proposition 5.3, we get a proof of Theorem 5.1 in the special case where $S_{1}=\emptyset$. We shall therefore assume, for the rest of this section, that $S_{1}$ is nonempty.

Proposition 5.4. There exists a rank one-idempotent $P \in \mathbb{M}_{2}$ such that

$$
\theta\left(S_{1}\right) \subseteq \overline{\mathbb{B}}_{P}^{\mathrm{HS}}(12 \delta) \sqcup \overline{\mathbb{B}}_{I-P}^{\mathrm{HS}}(12 \delta) .
$$

Moreover, given e, $f \in S_{1}$ :

- if $\widetilde{e}, \widetilde{f}$ both lie in $\overline{\mathbb{B}}_{P}^{\mathrm{HS}}(12 \delta)$, then so does $\widetilde{e f}$;

- if $\widetilde{e}, \widetilde{f}$ both lie in $\overline{\mathbb{B}}_{I-P}^{\mathrm{HS}}(12 \delta)$, then so does $\widetilde{e f}$;

- if $\widetilde{e} \in \overline{\mathbb{B}}_{P}^{\mathrm{HS}}(12 \delta)$ and $\widetilde{f} \in \overline{\mathbb{B}}_{I-P}^{\mathrm{HS}}(12 \delta)$, then ef $\in S_{0}$. 
The proof of Proposition 5.4 requires some work, which we break up into several lemmas. The first of these is an 'approximate version' of the following observation: if $P$ and $Q$ are rank-one idempotents in $\mathbb{M}_{2}$ with $P Q=0=Q P$, then $P+Q=I$.

This is also the first place where it is really necessary to make $\delta$ no bigger than about 0.03 , as we need to apply Lemma 5.2 to something which is 'approximately idempotent to within roughly $7 \delta^{\prime}$.

Lemma 5.5 (Separation lemma). Let e, $f \in S_{1}$ with ef $\in S_{0}$. Then

$$
\|\widetilde{e}+\widetilde{f}-I\|_{\mathrm{HS}} \leq 10 \delta \text { and }\|\widetilde{e}-\widetilde{f}\|_{\mathrm{HS}} \geq \frac{4}{3}-10 \delta>1 .
$$

Proof. Let $B=\widetilde{e}+\widetilde{f}$. We wish to prove that $B$ is close to $I$; this will follow if we can show that $B$ is approximately idempotent and has trace close to 2 .

Since $e f \in S_{0}$, applying Lemma 5.2(b) to the matrix $\widetilde{e f}$, with $\varepsilon=\delta$, yields $\|\widetilde{e f}\|_{\mathrm{HS}} \leq$ $\kappa(\delta) \delta$. Then, since

$$
\|\widetilde{e} \widetilde{f}+\widetilde{f} \widetilde{e}\|_{\mathrm{HS}} \leq\|\widetilde{e} \widetilde{f}-\widetilde{e f}\|_{\mathrm{HS}}+\|\widetilde{f} \widetilde{e}-\widetilde{e f}\|_{\mathrm{HS}}+2\|\widetilde{e f}\|_{\mathrm{HS}} \leq 2 \delta+2 \kappa(\delta) \delta,
$$

we obtain

$$
\left\|B^{2}-B\right\|_{\mathrm{HS}} \leq\left\|\widetilde{e}^{2}-\widetilde{e}\right\|_{\mathrm{HS}}+\left\|\widetilde{f}^{2}-\widetilde{f}\right\|_{\mathrm{HS}}+\|\widetilde{e} \widetilde{f}+\widetilde{f} \widetilde{e}\|_{\mathrm{HS}} \leq(4+2 \kappa(\delta)) \delta .
$$

As $(4+2 \kappa(\delta)) \delta \leq 6.1 \delta<0.183<2 / 9$, we may apply Lemma 5.2 (b) to the matrix $B$. By our earlier observation (5.7),

$$
|\operatorname{Tr}(B)-2| \leq|\operatorname{Tr}(\widetilde{e})-1|+|\operatorname{Tr}(\widetilde{f}-1)|<0.05+0.05<\frac{1}{2},
$$

and so (by Lemma 5.2(b)),

$$
\|B-I\|_{\mathrm{HS}} \leq \kappa(6.1 \delta) 6.1 \delta \leq \kappa\left(\frac{3}{4^{2}}\right) 6.1 \delta \leq\left(1-\frac{5}{14}\right)^{-1} 6.1 \delta \leq 10 \delta . \quad(* * *)
$$

For the second part, we apply the estimate (5.4) to obtain

$$
\|2 \widetilde{e}-I\|_{\mathrm{HS}} \geq(2-6 \delta)^{1 / 2} \geq \sqrt{1.82}>\frac{4}{3} .
$$

Combining this with $(* * *)$ yields

$$
\|\widetilde{e}-\widetilde{f}\|_{\mathrm{HS}}=\|(2 \widetilde{e}-I)-(B-I)\|_{\mathrm{HS}} \geq \frac{4}{3}-10 \delta>1,
$$

as required.

Intuitively, we should have $S_{1} \cdot S_{1} \subseteq\left(S_{1} \cup S_{0}\right)$ since elements of $\theta\left(S_{k}\right)$ are close to idempotents of rank $k$. Some care is needed to show this, because we have no a priori upper bound on norms of elements in $\theta\left(S_{1}\right)$.

Lemma 5.6. (i) If $e \in S, f \in S_{2}$ and $e \geq f$, then $e \in S_{2}$.

(ii) $S_{1} \cdot S_{1} \subseteq S_{1} \cup S_{0}$. 
Proof. Let $e, f \in S$ with $e f=f$. Then $\|\widetilde{e} \widetilde{f}-\widetilde{f}\|_{\mathrm{HS}} \leq \delta$. If $f \in S_{2}$, then just as in the proof of Proposition 5.3, we have $\left\|\widetilde{f}^{-1}\right\|_{\text {HS }}<1.5$. Hence, by Cauchy-Schwarz,

$$
|\operatorname{Tr}(\widetilde{e}-I)| \leq\|\widetilde{e} \widetilde{f}-\widetilde{f}\|_{\mathrm{HS}}\left\|\widetilde{f}^{-1}\right\|_{\mathrm{HS}} \leq 1.5 \delta \ll \frac{1}{2},
$$

forcing $e$ to lie in $S_{2}$. This proves (i).

Now if $e, g \in S_{1}$, put $f=e g$; by part (i), $f \notin S_{2}$, and (ii) is proved.

To analyse $S_{1}$ in further detail, the following lemma is useful.

Lemma 5.7 (Chains in $S_{1}$ ). Let $e, f \in S_{1}$ with $e \geq f$. Then $\|\widetilde{e}-\widetilde{f}\|_{\mathrm{HS}} \leq 5 \delta$.

Proof. Put $A=\widetilde{e}-\widetilde{f}$. We show that $A$ is approximately idempotent and has small trace, so must be close to 0 by Lemma 5.2(b). In detail: observe that

$$
\left\|A^{2}-A\right\|_{\mathrm{HS}}=\left\|\widetilde{e}^{2}-\widetilde{e}+\widetilde{f}^{2}-\widetilde{f}-\widetilde{e} \widetilde{f}+\widetilde{f}-\widetilde{f} \widetilde{e}+\widetilde{f}\right\|_{\mathrm{HS}} \leq 4 \delta<0.12,
$$

and $\operatorname{Tr}(A) \leq|1-\operatorname{Tr} \widetilde{e}|+|\underline{1}-\operatorname{Tr} \widetilde{f}| \leq 0.05+0.05 \ll 0.5$. Applying Lemma 5.2 (b) to the matrix $A$, we have $\|\widetilde{e}-\widetilde{f}\|_{\mathrm{HS}} \leq \kappa(4 \delta) 4 \delta \leq \kappa(0.12) 4 \delta$.

Calculation shows that

$$
\begin{aligned}
1-\kappa(0.12)^{-1} & =\sqrt{2} \rho(0.12) 0.12 \\
& =\frac{1}{\sqrt{2}}(1-\sqrt{1-0.48}) \\
& =\frac{1}{\sqrt{2}}-\sqrt{0.26}<0.2,
\end{aligned}
$$

so that $4 \kappa(0.12)<4(1-0.2)^{-1}=5$. The rest follows.

Proof of Proposition 5.4. Recall that $S_{1}$ is, by assumption, nonempty. Consider the relation on $S_{1}$ defined by $\left\{(e, f) \in S_{1} \times S_{1}: e f \in S_{1}\right\}$, and denote it by $\sim$. Clearly $\sim$ is symmetric and reflexive. We will see shortly that it is also transitive, as a consequence of the following two observations.

(i) If $e \sim f$ then $\|\widetilde{e}-\widetilde{f}\|_{\mathrm{HS}} \leq 10 \delta$. (For if $e, f$, ef $\in S_{1}$ then Lemma 5.7 implies that $\|\widetilde{e}-\widetilde{e f}\|_{\mathrm{HS}} \leq 5 \delta$ and $\|\widetilde{f}-\widetilde{e f}\|_{\mathrm{HS}} \leq 5 \delta$.)

(ii) If $e, f \in S_{1}$ and $\|\widetilde{e}-\widetilde{f}\|_{\mathrm{HS}} \leq 1$, then $e \sim f$. (This is immediate from the contrapositive of the separation lemma (Lemma 5.5).)

Therefore, since $20 \delta<1$, we see that $\sim$ is indeed transitive, and so $\sim$ is an equivalence relation on $S_{1}$.

Now let $e, f, g \in S_{1}$. Suppose that $e \nsim f$ and $f \nsim g$. Then, since $e f \in S_{0}$ and $f \underline{g} \in S_{0}$, the separation lemma (Lemma 5.5) implies that $\|\widetilde{e}+\widetilde{f}-I\|_{\mathrm{HS}} \leq 10 \delta$ and $\|\tilde{f}+\widetilde{g}-I\|_{\mathrm{HS}} \leq 10 \delta$. Hence

$$
\|\widetilde{e}-\widetilde{g}\|_{\mathrm{HS}} \leq\|\widetilde{e}+\widetilde{f}-I\|_{\mathrm{HS}}+\|\widetilde{f}+\widetilde{g}-I\|_{\mathrm{HS}} \leq 20 \delta \leq 0.6
$$

so by (ii), $e \sim g$. Thus there are at most two equivalence classes for this relation. 
Now, fix $p_{0} \in S_{1}$. By (i),

$$
\theta\left(\left[p_{0}\right]\right) \subseteq \overline{\mathbb{B}}_{\widetilde{p}_{0}}^{\mathrm{HS}}(10 \delta),
$$

where $\left[p_{0}\right]$ denotes the equivalence class of $p_{0}$ in $S_{1}$. Moreover, if $e \in S_{1} \backslash\left[p_{0}\right]$ then $e p_{0} \in S_{0}$ (by definition of $\sim$ ). Hence, by the separation lemma (Lemma 5.5), $\left\|\widetilde{e}+\widetilde{p}_{0}-I\right\|_{\mathrm{HS}} \leq 10 \delta$, so that

$$
\theta\left(S_{1} \backslash\left[p_{0}\right]\right) \subseteq \overline{\mathbb{B}}_{I-\widetilde{p}_{0}}^{\mathrm{HS}}(10 \delta) .
$$

By Lemma 5.2(b), there exists a rank-one idempotent $P \in \mathbb{M}_{2}$ such that $\left\|P-\widetilde{p}_{0}\right\|_{\mathrm{HS}} \leq 1.05 \delta$. Then

$$
\theta\left(\left[p_{0}\right]\right) \subseteq \overline{\mathbb{B}}_{P}^{\mathrm{HS}}(10 \delta+1.05 \delta) \subseteq \overline{\mathbb{B}}_{P}^{\mathrm{HS}}(12 \delta
$$

and

$$
\theta\left(S_{1} \backslash\left[p_{0}\right]\right) \subseteq \overline{\mathbb{B}}_{I-P}^{\mathrm{HS}}(10 \delta+1.05 \delta) \subseteq \overline{\mathbb{B}}_{I-P}^{\mathrm{HS}}(12 \delta) .
$$

Note that since $\|2 P-I\|_{\mathrm{HS}} \geq \sqrt{2}$ (by Lemma 5.2(b)) and $24 \delta \ll \sqrt{2}$, the sets $\theta\left(\left[p_{0}\right]\right.$ ) and $\theta\left(S_{1} \backslash\left[p_{0}\right]\right)$ are disjoint. Therefore,

$$
S_{1} \cap \theta^{-1}\left(\overline{\mathbb{B}}_{P}^{\mathrm{HS}}(12 \delta)\right)=\left[p_{0}\right] \quad \text { and } \quad S_{1} \cap \theta^{-1}\left(\overline{\mathbb{B}}_{I-P}^{\mathrm{HS}}(12 \delta)\right)=S_{1} \backslash\left[p_{0}\right] .
$$

Finally, let $e, f \in S_{1}$.

- If $\widetilde{e}, \widetilde{f} \in \overline{\mathbb{B}}_{P}^{\mathrm{HS}}(12 \delta)$ then $e \sim p_{0} \sim f$, so that $e f \sim p_{0}$ (as equivalence classes are closed under multiplication) and therefore $\widetilde{e f} \in \overline{\mathbb{B}}_{P}^{\mathrm{HS}}(12 \delta)$.

- If $\widetilde{e}, \widetilde{f} \in \overline{\mathbb{B}}_{I-P}^{\mathrm{HS}}(12 \delta)$, then $e \nsim p_{0}, f \nsim p_{0}$; since there are at most two equivalence classes, $e \sim f$. Thus $e \sim e f \sim f$, so $e f \nsim p_{0}$, so $\widetilde{e f} \in \overline{\mathbb{B}}_{I-P}^{\mathrm{HS}}(12 \delta)$.

- If $\widetilde{e} \in \overline{\mathbb{B}}_{P}^{\mathrm{HS}}(12 \delta)$ and $\widetilde{f} \in \overline{\mathbb{B}}_{I-P}^{\mathrm{HS}}(12 \delta)$, then $e \sim p_{0}$ and $p_{0} \nsim f$. Thus $e \nsim f$, so ef $\in S_{0}$.

This completes the proof of the proposition.

We now fix an idempotent $P \in \mathbb{M}_{2}$ that satisfies the conclusions of Proposition 5.4. The sets

$$
\overline{\mathbb{B}}_{I}^{\mathrm{HS}}(2 \delta), \quad \overline{\mathbb{B}}_{P}^{\mathrm{HS}}(12 \delta), \quad \overline{\mathbb{B}}_{I-P}^{\mathrm{HS}}(12 \delta), \quad \overline{\mathbb{B}}_{0}^{\mathrm{HS}}(2 \delta)
$$

are pairwise disjoint, and their union contains $\theta(S)$. Recall that we have already defined $\phi: S_{2} \sqcup S_{0} \rightarrow \mathbb{M}_{2}$ which is multiplicative; now define $\phi: S_{1} \rightarrow \mathbb{M}_{2}$ by setting $\phi(x)$ to be whichever of $P$ and $I-P$ is closer to $\widetilde{x}$. Explicitly, the map $\phi: S \rightarrow \mathbb{M}_{2}$ satisfies:

$$
\phi(x)= \begin{cases}I & \text { if } x \in S_{2}, \\ P & \text { if } \widetilde{x} \in \overline{\mathbb{B}}_{P}^{\mathrm{HS}}(12 \delta), \\ I-P & \text { if } \widetilde{x} \in \overline{\mathbb{B}}_{I-P}^{\mathrm{HS}}(12 \delta), \\ 0 & \text { if } x \in S_{0} .\end{cases}
$$

By construction, $\|\widetilde{x}-\phi(x)\|_{\mathrm{HS}} \leq 12 \delta$ for all $x \in S$. 
It remains to show that $\phi$ is multiplicative. Let

$$
S_{p}:=\theta^{-1}\left(\overline{\mathbb{B}}_{P}^{\mathrm{HS}}(12 \delta)\right) \quad \text { and } \quad S_{q}:=\theta^{-1}\left(\overline{\mathbb{B}}_{I-P}^{\mathrm{HS}}(12 \delta)\right) .
$$

Then $S_{1}=S_{p} \sqcup S_{q}$, and it suffices to verify the following claims:

(1) $S_{2} \cdot S_{2} \subseteq S_{2}$;

(2) $S \cdot S_{0} \subseteq S_{0}$;

(3) $S_{2} \cdot S_{p} \subseteq S_{p}$ and $S_{2} \cdot S_{q} \subseteq S_{q}$;

(4) $S_{p} \cdot S_{p} \subseteq S_{p}$ and $S_{q} \cdot S_{q} \subseteq S_{q}$;

(5) $S_{p} \cdot S_{q} \subseteq S_{0}$.

Assertions (1) and (2) follow from Proposition 5.3. Assertions (4) and (5) follow from Proposition 5.4. Assertion (3) requires some more work, and is dealt with in our final proposition.

Proposition 5.8. Let $e \in S_{2}$. If $f \in S_{p}$, then so is ef; if $f \in S_{q}$, then so is ef.

Remark 5.9. Although we know that $\widetilde{e}$ is close to $I$ for each $e \in S_{2}$, there might still exist rank-one idempotents $R \in \mathbb{M}_{2}$ such that $\|\widetilde{e} \cdot R-R\|$ is large. So while the conclusion of Proposition 5.8 is as one would expect, the proof is somewhat circuitous.

Proof of Proposition 5.8. Let $e \in S_{2}$ and $f \in S_{1}$. By Lemma 5.6, ef $\notin S_{2}$. We claim that $e f \notin S_{0}$. Assume that $e f \in S_{0}$; then by Lemma 5.2(b), $\|\widetilde{e f}\|_{\mathrm{HS}} \leq \kappa(\delta) \delta \leq 1.05 \delta$. Since $e \in S_{2}$, we have $\left\|(\widetilde{e})^{-1}\right\|_{\mathrm{HS}}<1.5$, by the same argument as in the proof of Proposition 5.3. Therefore, by Cauchy-Schwarz,

$$
|\operatorname{Tr} \widetilde{f}| \leq\left\|(\widetilde{e})^{-1}\right\|_{\mathrm{HS}}\|\widetilde{e} \widetilde{f}\|_{\mathrm{HS}} \leq 1.5\left(\delta+\|\widetilde{e f}\|_{\mathrm{HS}}\right) \ll 0.95 .
$$

But, by (5.8), this implies that $f \in S_{0}$, contradicting the assumption that $f \in S_{1}$.

The only remaining possibility is that $e f \in S_{1}$, and hence, by Lemma 5.7,

$$
\|\widetilde{f}-\widetilde{e f}\|_{\mathrm{HS}} \leq 5 \delta \leq 0.15 \text {. }
$$

On the other hand, recalling that $\|2 P-I\|_{\mathrm{HS}} \geq \sqrt{2}$ (by Lemma 5.2(b)), we see that the distance between $\overline{\mathbb{B}}_{P}^{\mathrm{HS}}(12 \delta)$ and $\overline{\mathbb{B}}_{I-P}^{\mathrm{HS}}(12 \delta)$ is bounded below by $\sqrt{2}-24 \delta>0.6$. Therefore, $\widetilde{f}$ and $\widetilde{e f}$ either both belong to $\theta\left(S_{p}\right)$ or both belong to $\theta\left(S_{q}\right)$. This completes the proof.

Since Proposition 5.8 implies assertion (3), the function $\phi: S \rightarrow \mathbb{M}_{2}$ is indeed multiplicative, and this completes the proof of Theorem 5.1.

\section{Concluding remarks and questions}

It would be interesting to try and find an intrinsic condition on a semilattice which is necessary and sufficient for the existence of some weight $\omega$ such that $\ell_{\omega}^{1}(S)$ is not AMNM. We have seen (Example 3.13(ii) and Theorem 3.14) that $b(S)=+\infty$ is 
a necessary condition; it may also be a sufficient condition, although we have not investigated further.

As remarked after the proof of Theorem 4.7, one can build on Theorem 5.1 to show

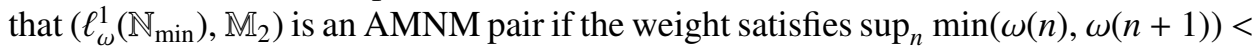
$\infty$. Details will be given in forthcoming work, which also plans to address AMNM pair problems for $\left(\ell^{1}(S), B\right)$, with $B$ an arbitrary Banach algebra. There is some evidence to suggest that the method used for $B=\mathbb{M}_{2}$ can be extended to $B=\mathbb{M}_{n}, n \geq 3$, although a more laborious case-by-case analysis would be required. (However, see Remark 1.2.)

We close with the following question: is $\left(\ell^{1}(S), \mathcal{B}(E)\right)$ AMNM for every Banach space $E$ ? If not, what if we restrict to the cases $E=\ell^{p}$ for $1<p<\infty$ ?

\section{Acknowledgements}

Some parts of this paper are based on work done in 2011, during a visit to Newcastle University. The author thanks the School of Mathematics and Statistics at the University for their hospitality. Thanks also go to the anonymous referee for his or her diligent work, which caught several minor errors, led to the correct formulation of Theorem 4.7, and contained valuable suggestions that have improved the paper. The work here was supported by NSERC Discovery Grant 402153-2011. Figure 1 was produced using Paul Taylor's diagrams. sty macros.

\section{References}

[1] G. Birkhoff, Lattice Theory, 3rd edn, American Mathematical Society Colloquium Publications, 25 (American Mathematical Society, Providence, RI, 1979).

[2] H. G. Dales and R. J. Loy, 'Approximate amenability of semigroup algebras and Segal algebras', Dissertationes Math. (Rozprawy Mat.) 474 (2010), 58.

[3] J. F. Feinstein, 'Strong Ditkin algebras without bounded relative units', Int. J. Math. Math. Sci. 22(2) (1999), 437-443.

[4] F. Ghahramani, R. J. Loy and Y. Zhang, 'Generalized notions of amenability, II', J. Funct. Anal. 254(7) (2008), 1776-1810.

[5] E. Hewitt and H. S. Zuckerman, 'The $l_{1}$-algebra of a commutative semigroup', Trans. Amer. Math. Soc. 83 (1956), 70-97.

[6] R. A. J. Howey, 'Approximately multiplicative maps between some Banach algebras', $\mathrm{PhD}$ thesis, The University of Newcastle-upon-Tyne, 2000.

[7] R. A. J. Howey, 'Approximately multiplicative functionals on algebras of smooth functions', J. Lond. Math. Soc. (2) 68(3) (2003), 739-752.

[8] K. Jarosz, Perturbations of Banach Algebras, Lecture Notes in Mathematics, 1120 (Springer, Berlin, 1985).

[9] K. Jarosz, 'Almost multiplicative functionals', Studia Math. 124(1) (1997), 37-58.

[10] B. E. Johnson, 'Approximately multiplicative functionals', J. Lond. Math. Soc. (2) 34(3) (1986), 489-510.

[11] B. E. Johnson, 'Approximately multiplicative maps between Banach algebras', J. Lond. Math. Soc. (2) 37(2) (1988), 294-316.

[12] J. Lawrence, 'The stability of multiplicative semigroup homomorphisms to real normed algebras. I', Aequationes Math. 28(1-2) (1985), 94-101. 
[13] N.N., 'personal communication', http://mathoverflow.net/questions/100971 (version: 2012-0629), MathOverflow, 2012.

[14] S. J. Sidney, 'Are all uniform algebras AMNM?', Bull. Lond. Math. Soc. 29(3) (1997), 327-330.

YEMON CHOI, Department of Mathematics and Statistics, McLean Hall, University of Saskatchewan, 106 Wiggins Road, Saskatoon, SK, Canada S7N 5E6 e-mail: choi@math.usask.ca 